\title{
ROYAL ASIATIC SOCIETY OF
}

\section{GREAT BRITAIN \& IRELAND (FOUNDED MARCH, 1823)}

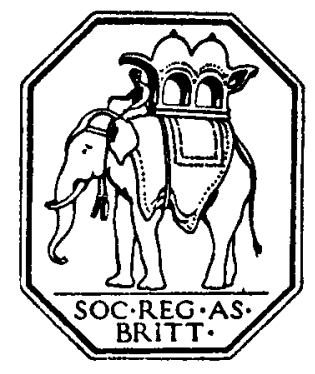

\section{LIST OF FELLOWS,}

LIBRARY ASSOCIATES AND SUBSCRIBERS

1979

PUBLISHED BY THE SOCIETY

56 QUEEN ANNE STREET LONDON WIM 9LA 
56 QUEEN ANNE STREET, LONDON, WIM 9LA

(Tel.: 01-935 8944)

Patron

HER MOST EXCELLENT MAJESTY THE QUEEN

Vice-Patron

HIS ROYAL HIGHNESS THE PRINCE OF WALES

Honorary Vice-Presidents

1963 PROFESSOR SIR RALPH L. TURNER, MC

1976 PROFESSOR SIR HAROLD BAILEY

1976 PROFESSOR E. J. W. SIMON, CBE

COUNCIL OF MANAGEMENT FOR 1979-80

1979 PROFESSOR SIR CYRIL PHILIPS President

Director

1977 MR D. J. DUNCANSON, OBE

Vice-Presidents

1976 PROFESSOR E. H. S. SIMMONDS

1977 PROFESSOR K. A. BALLHATCHET

1978 MR E. P. SOUTHALL

1979 PROFESSOR C. F. BECKINGHAM

1978 MR G. A. CALVER (Hon. Treasurer)

Honorary Officers

1977 MR N. M. LOWICK (Hon. Secretary)

1971 MR S. E. DIGBY (Hon. Librarian)

1979 DR A. D. H. BIVAR (Hon. Editor)

1978 MAJOR J. E. BARWIS-HOLLIDAY

Ordinary Members of Council

1976 MR A. S. BENNELL

1979 MR R. M. BURRELL

1976 MR J. BURTON-PAGE

1979 MR A. H. CHRISTIE

1976 PROFESSOR C. J. DUNN

1977 MR J. F. FORD, CMG, OBE

1978 MR F. G. GOODWIN

1979 DR M. A. N. LOEWE

1976 MR P. S. MARSHALL

1979 PROFESSOR V. L. MÉNAGE

1977 MR B. W. ROBINSON

1979 THE RT HON THE EARL OF SELKIR K, KT, PC, GCMG, GBE, AFC, QC

1977 DR S. A. SKILLITER

1979 DR L. L. WARE

\section{Secretary}

1978 MISS E. V. GIBSON

Librarian

1978 DR R. PANKHURST

Honorary Solicitors

MESSRS PHILIP CONWAY THOMAS \& CO 


\section{PRESIDENTS OF THE SOCIETY SINCE ITS FOUNDATION}

1823 RT. HON. CHARLES WATKIN WILLIAMS WYNN, M.P.

1841 RT. HON. THE EARL OF MUNSTER.

1842 RT. HON. THE LORD FITZGERALD AND VESEY.

1843 RT. HON. THE EARL OF AUCKLAND.

1849 RT. HON. THE EARL OF ELLESMERE.

1852 RT. HON. THE EARL ASHBURTON.

1855 PROFESSOR HORACE HAYMAN WILSON.

1859 COLONEL WILLIAM HENRY SYKES, M.P.

1861 RT. HON. THE VISCOUNT STRANGFORD.

1864 SIR THOMAS EDWARD COLEBROOKE, BART., M.P.

1867 RT. HON. THE VISCOUNT STRANGFORD.

1869 SIR T. E. COLEBROOKE, BART.

1869 MAJOR-GENERAL SIR HENRY CRESWICKE RAWLINSON, BART.

1871 SIR T. E. COLEBROOKE, BART.

1872 SIR HENRY BARTLE EDWARD FRERE.

1875 SIR T. E. COLEBROOKE, BART.

1878 MAJOR-GENERAL SIR H. C. RAWLINSON, BART.

1881 SIR T. E. COLEBROOKE, BART.

1882 SIR H. B. E. FRERE.

1884 SIR WILLIAM MUIR.

1885 COLONEL SIR HENRY YULE.

1887 SIR THOMAS FRANCIS WADE.

1890 R T. HON. THE EARL OF NORTHBROOK.

1893 RT. HON. THE LORD REAY.

1921 LIEUT.-COL. SIR RICHARD CARNAC TEMPLE, BART.

1922 RT. HON. THE LORD CHALMERS

1925 SIR EDWARD D. MACLAGAN.

1928 THE MOST HON. THE MARQUESS OF ZETLAND.

1931 SIR EDWARD D. MACLAGAN.

1934 PROFESSOR D. S. MARGOLIOUTH.

1937 RT. HON. THE LORD HAILEY.

1939 THE MOST HON. THE MARQUESS OF WILLINGDON.

1940 RT. HON. THE VISCOUNT SAMUEL.

1943 SIR RICHARD WINSTEDT.

1946 RT. HON. THE EARL OF SCARBROUGH.

1949 SIR RICHARD WINSTEDT.

1952 PROFESSOR SIR RALPH TURNER.

1955 SIR RICHARD WINSTEDT.

1958 SIR GERARD CLAUSON.

1961 SIR RICHARD WINSTEDT.

1964 PROFESSOR SIR HAROLD BAILEY.

1967 PROFESSOR C. F. BECKINGHAM.

1970 B. W. ROBINSON, ESQ.

1973 PROFESSOR E. H. S. SIMMONDS.

1976 PROFESSOR C. F. BECKINGHAM.

1979 PROFESSOR SIR CYRIL PHILIPS. 


\section{FOREIGN EXTRAORDINARY FELLOWS}

1971 H. E. Zeki Küneralp

1975 H. I. H. Prince Mikasa of Japan
1979 H. E. Phan Wannamathee

\section{HONORARY FELLOWS}

1952 Prof. W. Norman Brown, Philadelphia.

1954 Dr. Jean Deny, France.

1954 Dr. Sushil Kumar De, Calcutta.

1954 Prof. Dr. J. Gonda, Utrecht.

1955 Prof. S. Elisséef, Cambridge, Mass.

1957 Dr. R. C. Majumdar, Calcutta.

1958 Prof. K. A. Nilakanta Sastri, Madras.

1958 Prof. C. F. A. Schaeffer, Paris.

1958 Prof. Dr. Ernst Waldschmidt, Göttingen.

1961 Prof. J. Pederson, Copenhagen.

1962 Prof. I. M. Diakonoff, Leningrad.

1964 Rev. Prof. E. Lamotte, Louvain.

1967 Prof. V. I. Abaev, Moscow.

1967 Prof. Louis Ligeti, Budapest.

1969 Prof. M. B. Emeneau, Berkeley, California.

1969 H. E. Dr. Enrico Cerulli, Rome.

1969 Prof. Dr. Annemarie von Gabain, Anger.
1970 Prof. H. J. Polotsky, Jerusalem.

1971 H. E. Dr. Gunnar Jarring, Sweden.

1975 Prof. Nikolay Aleksandrovich Baskakov, Moscow.

1975 Prof. C. H. Gordon, Brookline.

1975 Prof. Andrey N. Kononov, Leningrad.

1975 Dr. C. Sivaramamurti, New Delhi.

1975 Prof. G. Tucci, Rome.

1978 Prof. Paul Balog, Rome.

1978 Prof. J. Blau, Jerusalem.

1978 Prof. C. Cahen, France.

1978 Prof. K. Enoki, Tokyo.

1978 Prof. D. E. Hettiaratchi, Sri Lanka.

1978 Prof. H. Inalcik, Chicago.

1978 Prof. Dr. T. Lewicki, Krakow.

1978 Prof. H. Nakamura, Tokyo.

1978 Prof. H. K. Sherwani, Hyderabad.

\section{FELLOWS HONORIS CAUSA}

1975 Mr. G. A. Calver

1978 Miss M. D. C. Crawford

1975 Dr. J. F. Hansman

\section{GOLD MEDALLISTS}

N.B. - The Gold Medal was founded in 1897.

1897 Prof. E. B. Cowell.

1900 E. W. West.

1903 Sir William Muir.

1906 G. U. Pope.

1909 Sir G. A. Grierson.

1912 J. F. Fleet.

1915 Mrs. Agnes Smith Lewis.

1915 Mrs. Margaret Dunlop Gibson.

1918 V. A. Smith.

1922 Prof. H. A. Giles.

1925 Rev. A. H. Sayce.

1928 Prof. D. S. Margoliouth.

1932 Sir Aurel Stein.

1935 Sir E. Denison Ross.

1938 Prof. R. A. Nicholson.
1941 Prof. F.W. Thomas.

1944 Sir John Marshall.

1947 Sir Richard Winstedt.

1950 Dr. L. D. Barnett.

1953 Sir Ralph Turner.

1956 Prof. W. Perceval Yetts.

1959 Prof. K. A. C. Creswell.

1962 Prof. V. Minorsky.

1965 Prof. G. H. Luce.

1968 Sir Hamilton Gibb.

1971 Sir Harold W. Bailey.

1973 Sir Gerard L. M. Clauson (Sesquicentenary Commemorative Medal).

1977 Prof. W. Simon. 


\section{BURTON MEMORIAL MEDALLISTS}

N.B.-The Medal was founded in 1923.

1925 H. St. J. B. Philby.

1928 Sir Harold A. MacMichael.

1931 Bertram S. Thomas.

1934 Miss Freya Stark.

1937 Sir Arnold T. Wilson.

1940 Major J. B. Glubb.

1943 W. H. Ingrams.

1947 Col, and Mrs. D. L. R. Lorimer.
1952 Miss G. Caton Thompson.

1963 C. J. Edmonds.

1966 W. P. Thesiger.

1969 Brigadier Stephen Longrigg.

1972 Professor Mary Boyce.

1978 Dr. W. G. Archer.

1978 Dr. H. T. Lambrick.

\section{BRANCH AND ASSOCIATE SOCIETIES}

The Asiatic Society, 1 Park Street, Calcutta.

The Asiatic Society of Bombay (1838).

The Sri Lanka Branch of the Royal Asiatic Society (1845).

The Korean Branch of the Royal Asiatic Society (1900).

The Malaysian Branch of the Royal Asiatic Society (1878).

The Asiatic Society of Japan (1875).

The Bihar and Orissa Research Society.

The Burma Research Society.

The Madras Literary Society and Auxiliary of the R.A.S.

The Mythic Society, Bangalore.

The Hong Kong Branch of the Royal Asiatic Society. 


\section{LIST OF FELLOWS \\ AND LIBRARY ASSOCIATES}

F.E.F. = Foreign Extraordinary Fellow.

$\dagger=$ Fellows who have compounded for their subscriptions.

$\ddagger=$ Library Associates.

$\S=$ Fellows who have served on the Council.

HER MOST EXCELLENT MAJESTY THE QUEEN

HIS ROYAL HIGHNESS THE PRINCE OF WALES

1957 AALTO, Prof. P., Hollantilaisentie 1. Helsinki 10330.

HON. 1967 ABAEV, Prof. V. I., Volkhonka 18, Moscow.

1925 † ABIDI, Major S. M. A. H. H., Nisheman, Amraoti Camp (2), Berar, India.

1970 ABOU-EL-HAJ, Dr. R., 516 Midvale Avenue, Los Angeles, Cal. 90024.

1978 ABRAHAM, V. B.S., 45 Stanley Park Road, Carshalton, Surrey.

1979 ABRAHAM, Dr. V. C., Dept. of Health, Government of Kuwait, P.O. Box 400, Kuwait.

1979 ADAMS, Prof. D.K., 152 S. Clark Drive, Beverley Hills, Cal. 90211.

1966 †ADAMSON, P. B., West View Lodge, 6 Crag Lane, Knaresborough, North Yorks.

1979 AfZal Ul-HUQ, Prof. Dr. Seyd, P.O. Box 1247, Sana'a, Yemeni Arab Republic.

1967 AGA KHAN, H. H. Prince Sadruddin, Chateau de Bellerive, Collonge-Bellerive, Geneva.

1973 AGARWAL, Dr. C. P., Kedarnath Street, Senapat, Farrukhabad, India.

1959 † AGGARWAL, Dr. H. P. C., Shri Bhichha Mal Building, 115/a Kamla Nagar, Delhi 7.

1968 AGGARWAL, Mrs. M., "Constanceville", 11, Faircross Avenue, Barking, Essex.

1977 AHMAD, N., 49 Erskine Street, Aberdeen.

1975 AHMAD, S. M., Centre of West Asian Studies, Aligarh Muslim University, Aligarh, U.P., India.

1977 AIKEN, W. A. K., P.O. Box 10352, Greenville, S. Carolina.

1975 AITKEN, Miss B. M. W., 11 Beasley Gardens, Lansdown Row, Cheltenham.

1976 AIY AR, S. A., 49 Apollo Street, Bombay 1.

1976 AKAILVI, S. N. M., 532, Hyde Park Gate, London S.W.7.

1967 ALDER, Dr. C., la Bennett Park, London S.E.3.

1968 AlLAN, J. W., Ashmolean Museum, Oxford.

1953 §ALLCHIN, F. R., West Gate House, Barrington, Cambridge.

1960 ALlOTT, Mrs. A. J., 21 Windsor Road, London N.3.

1976 ALON, Dr. I., Dept. of Islamic Civilization, The Hebrew University, Jerusalem.

1974 AL-RASHID, S. A., Dept. of Semitic Studies, University of Leeds, Leeds.

1978 AL-RAWI, Dr. F. N. H., Archaeology Dept., Arts College, University of Baghdad, Baghdad.

1977 Al-ZAIDAN, A. A., Dept. of Semitic Studies, University of Leeds, Leeds.

1978 AMBROSE, A. F., 57 Fair Street, Broadstairs, Kent.

1979 ANDREWS, P. A., 74 Westbourne Terrace, London W.2.

1979 ANSARI, Prof. G. R., Government Ismailia College, Vihari, Pakistan.

1972 AOKI, Mrs. M. Y., 50 Follen Street, Cambridge, Mass. 02138.

1976 APPLEYARD, D. L., S.O.A.S., Malet Street, London WC1E 7HP.

1963 AQUILINA, Prof. J., Villa Rihana, Old Railway Road, Balzan, Malta.

1962 ARAFAT, Prof. W. N., Institute of Arabic and Islamic Studies, Cartmel College, Bailrigg, Lancaster.

1966 ARBUCKLE, Prof. J., Institute of Translators \& Interpreters, Laurentian University, Sudbury, Ontario, Canada.

1977 ARIS, M. V., St. John's College, Oxford OX1 3JP. 
ARMITAGE, Major H. St. J. B., The Old Vicarage, E. Horrington, Somerset BA5 3EA.

AROORAN, Dr. K. N., 3/9 Tank Bund Road, Nungambakham, Madras 600034.

ARORA, Dr. J. K., Patel Nagar, Railway Road, Hapur 245101, India.

$\dagger$ ARTHUR, C. D., P.O. Box 445, Paoli, Pa. 19301.

ARTOLA, Dr. G. T., University of Toronto, Dept. of East Asian Studies, Toronto 5 .

ASHER, F.M., Dept. of Art History, University of Minnesota, Minneapolis, Minnesota 55455.

$\dagger$ ASHER, R. E., Dept. of Linguistics, University of Edinburgh, Adam Ferguson Building, George Square, Edinburgh.

1964 ASMUSSEN, J. P., Bredgade 77, Copenhagen K, Denmark.

1971 AUCHTER LONIE, J. P., University Library, Bailrigg, Lancaster.

1963 AUSTIN, E. W., 33 Natal Road, London S.W.16.

1960 AVERY, P. W., King's College, Cambridge.

1977 AZAMI, C. A., 102 Sahba (Give) Street, Amirabad Street, Tehran.

1969 BACH, S. C. G., C.B.E., 11 Portland Road, Summertown, Oxford.

1966 BA-FAQIH, S. M., Ambassade de la République Démocratique du Yemen, 88 Boulevard Malesherbes, Paris 8.

BAILEY, G. E. J., 12 Shaftesbury Avenue, Kenton, Harrow, Middlesex.

$\dagger \S$ B AILEY, Prof. Sir Harold, Queens' College, Cambridge.

BAKALLA, Dr. M. H., Arabic Language Institute, University of Riyadh, PO Box 4274, Riyadh.

1954 BAKE, Mrs. C. M., 119 Endsleigh Court, Upper Woburn Place, London W.C.1.

1972

1970

1978

1966

1958

BAKER, Major A. D., Flat 2, 52 Kensington Court, London W.8.

BAKER, Mrs. P. L., 146 Cheyneys Avenue, Canons Park, Edgware, Middlesex.

BALAMBAL, Dr. V., Dept. of Indian History, University of Madras, Madras 600005 .

BALL, Rev. D., 2 Tower Mansions, 104 West End Lane, London N.W.6.

§BALlHatcheT, Prof. K. A., 11 The Mead, Clevelands, London W.13.

HON. 1978 B LINGAL, Miss C., 5 Logan Place, London W.8.

1973 †ANDYOPADYAY, Dr. S., Nila-Kamala, 203 Lake Town, Block A, Calcutta 55.

1978 BANERJEE, Prof. B., Dept. of Sanskrit, Pali 4, Prakrit, Viva Bharate University, Santiniketan 31235, West Bengal.

1966 BANERJEE, Pandit M. M. S., 43 Gopal Banerjee Lane, Howrah, West Bengal.

1965 BARgEBUHR, Prof. Dr. F. P., The Ecumenical Institute for Advanced Theological Studies, PO Box 19556, Tantur, Jerusalem.

1949 BARNeTT, K. M. A., O.B.E., UN Development Programme, P.O. Box 333, Zomba, Malawi.

1971 BARNETT, R. D., C.B.E., 14 Eldon Grove, London N.W.3.

1977 \$BARNETT, R. J., 14 Eldon Grove, London N.W.3.

1969 §BARR, Prof. J., 6 Fitzherbert Close, Iffley, Oxford.

1974 †BARRATT, Dr. G. R. V., Dept. of Russian, Carleton University, Ottawa.

1949 BARRETT, D. E., The Old Bakery, Drayton Parslow, Bletchley, Bucks.

1957 §BARRETT, E. C. G., C.M.G., Hillfield, Amlet's Lane, Cranleigh, Surrey.

1970 BARWIS-HOLLIDAY, Major J. E., Glydwish Hall, Burwash, E. Sussex TN19 7DG.

1963 BASHAM, Mrs. A. L., Australian National University, Dept. of Oriental Civilization, Canberra.

1948 §BASHAM, Prof. A. L., School of General Studies, Box 4, P.O. Canberra.

HON. 1975 BASKAKOV, Prof. N. A., 117333 Moscow V333, Ul.D.Ul'yanova 4/34, B.383. 
1975

1962

1939

1949

1968

1947

1954

1968

1949

1973

1974

1965

1935

1976

1966

1967

1973

1969

1977

1973

1960

1977

1968

1977

1975

1974

1946

1968

1978

1973

1978

1964

1974

1974

1970

1959

1934

HON.

1979

1964

1977

1965

1978

1963

1966

1960

1960

1975

1968

1946

1949

1953

1959

BASRI, M. S., 244 Dover House Road, London SW15 5DA.

BASSETT, Dr. D. K., The Centre for South East Asian Studies, University of Hull, Yorks.

BASTIN, R. W., Eastleigh, The Ridgeway, Oxshott, Surrey.

BATTERsBY, Prof. H. R., Box 80, Groveland, New York 14462.

BAUSANI, Prof. A., Coop. Buona Sorte, Via Filippo de Grenet-Tre Pini, 00128 Rome.

†BAVEJA, M. R., C-504 Defence Colony, New Delhi 3.

BAWDEN, Prof. C. R., Holwyn, 19 Richings Way, Iver, Bucks.

BECKER, Dr. M. J., 37 Hazelwell Road, London S.W.15.

BEC KINGHAM, Prof. C. F., 56 Queen Anne Street, London W1M 9LA.

BECKINGHAM, Mrs. E., 33 Causewayside, Fen Causeway, Cambridge.

BEDI, R. S., Magistrate First Class, Mokokchung 798601, Nagaland, India.

BEE, P. J., S.O.A.S., Malet Street, London WC1E 7HP.

§BEESTON, Prof. A. F. L., St. John's College, Oxford.

BEG, Dr. M. A. J., Universiti Kebangsaan Malaysia, Kuala Lumpur.

BEGUM, Mrs. G., J.P., 14B G.A.A.Khan, 3rd Street, Cathedral, Madras 6.

\$BELGRA VE, J. H. D., 11 Arrayedh Gardens, Manama, Bahrain, P.O. Box 551.

BELLINGHAM, J., The Cliffs, Baily, Co. Dublin.

BENDER, Prof. E., 5014 Woodbine Avenue, Philadelphia, Pa. 19131.

BENECKE, G., Darwin College, The University, Canterbury, Kent.

§BENNELL, A. S., 81A Ashley Road, Walton-on-Thames KT12 1HQ.

BHARATl, A. S. G., Shri Shri Shivakali Mandir, 7/1 Jessore Road, Calcutta 28.

BHATIA, Dr. K. C., National Academy of Administration, Mussourie, 248179 U.P. India.

BhatTA, Dr. P., E-7/7 Vasant Vihar, New Delhi 110057.

BHATNAGAR, Dr. G. D., Banaras Hindi University, Varanasi 5.

BHATTACHARYA, N. J., 98/6 Neogipara Road, Barahanagar, Calcutta 700036.

BHU R, Capt. R. A. O., Flat 10, 2 Russell Street, Calcutta 16.

BIBBY, T. G., Horretløkken, Marslet, Denmark.

BIDDLE, D. W., Beaver Hill, Box 150, Montague, Mass. 01351.

BIDDLE, Miss M. L., Beaver Hill, Box 150, Montague, Mass. 01351.

BIJUR, G., 65 Eaton Square, London S.W.1.

BILlingSLEY, A. J., Dept. of Foreign Affairs, Canberra.

BIRCH, I. J., South Cottage, Skircoat Green Road, Halifax HX3 0LB.

BIRDSALL, Dr. J. N., 70 Shutlock Lane, Birmingham 11.

§BIV AR, Dr. A. D. H., 127 Mayfield Avenue, London, N.12.

§BLACKER, Dr. C. Willow House, Grantchester.

†BLACKMORE, Dr. T. "Bay Trees", Padleigh Hill, Englishcombe, Bath, Avon.

†B LACKWOOD, Miss U. H., M.B.E., Craigowrie, Boat of Garten, Inverness-shire.

1978 BLAU, Prof. J., Dept of Arabic, The Hebrew University, Jerusalem.

BLOOM FIELD, B. C., 99 Morden Hill, London SE13 7NP.

BLUE, A. D., 12 Douglas Crescent, Edinburgh 12 5BB.

BLUNKETT, I. R., 45A, Worcester Road, Sutton, Surrey.

BODE, Prof. D. F. A., "Heera-Meher", Wodehouse Road, Colaba, Bombay 5.

BORDINI, M. A., Av. Luro 3363, 7600, Mar del Plata, Argentina.

BOSCARO, A., Via Doge D. Michiel 8, 30126 Lido di Venezia, Venice.

BOSTON, D. M., Horniman Museum, London Road, London S.E.23.

§BOSWORTH, Prof. C.E., 11 Prince's Road, Heaton Moor, Stockport, Cheshire.

BOTto, Prof. O., 97 Via Principessa Clotilde, Turin.

Boulting, F., 3 Brynmaer Road, London S.W.11.

§BOWNAS, Prof. G., Centre of Japanese Studies, The University, Sheffield 10.

$\dagger$ §BOXER, Prof. C. R., Ringshall End, Little Gaddesden, Berkhamsted.

$\dagger \S B O Y C E$, Prof. M., 30b Shepherds Hill, London N.6.

†BRAHMACHARI, B. N., 16 Pangali, Nutanganj, Burdwan 713 101, India.

BREDT, C., 50 Tenison Road, Cambridge CB1 2DW. 
1966

1963

1973

1971

1975

1946

1974

1945

1972

1959

1970

1960

HON.

1976

1976

1972

1977

1964

1970

1932

1960

1946

1971

BREEN, M. C., Flat 10, 294 Pacific Highway, Artarmon 2064, N.S.W., Australia. BRELVI, Prof. E., Dept. of Urdu, University of the Panjab, Lahore.

B REND, Miss B., 148 Haverstock Hill, London NW3 2AY.

BREWSTER, Rev. D. P., The Vicarage, Wilverley Road, Brockenhurst, Hants.

BRICE, W. C., Cherry Tree Cottage, 14 Barlow Moor Road, Manchester M20 8GJ.

BROOKES, Rev. Dr. R. S., B2 Calthorpe Mansions, Fiveways, Birmingham 15.

†BROOME, M. R., 30 Warren Road, Woodley, Berks.

BROUGH, Prof. J., 5 Thorn Grove, Bishop's Stortford, Herts CM23 5LB.

BROWN, C. H., 1104 Fleetwood Drive, Lookout Mountain, Tennessee 37350.

BROWN, Mrs. H. W., Ashmolean Museum, Oxford.

BROWN, R. Lamont, 34 Spottiswoode Gardens, St. Andrews, KY16 8SB.

BROWN, Mrs. M., Heathcote, 13 Beulah Road, Epping, Essex EM16 6RH.

1962 BROWN, Prof. W. N., University of Pennsylvania, Philadelphia 1.

BROWN, Mrs. Y.-Y., 105 Priory Road, London N8 8LY.

BROWNE, Miss A. M., 29 Pembroke Road, London W.8.

BRYER, D. R. W., 39 Portland Road, Oxford.

BUCHAN, J., 11 Mount Row, London W.1.

BUNFORD, Mrs. J., M.B.E., Flat 2, 39 Roland Gardens, London S.W.7.

§BUR RELL, R. M., S.O.A.S., Malet Street, London, WC1E 7HP.

§BUR ROW, Prof. T., 1 Woodlands, Kidlington, Oxford.

§BURTON-PAGE, J., Knowle Farm House, Church Knowle, Wareham, Dorset.

†BURTT, J. O., 102 Marine Avenue, Monkseaton, Northumberland.

BYNON, Dr. J. F. G., S.O.A.S., Malet Street, London, WC1E 7HP.

HON. 1978 CAHEN, Prof. C., 62 Avenue Carnot, Savigny/Orge, France.

1978 CAldWEll-Smith, Miss J., Flat 9, Clare Court, 2 Grosvenor Hill, London SW19 4RZ.

HON. CAUSA $1975+$ CAlver, G. A., Darband House, Blackdown Avenue, Pyrford, Nr. Woking, Surrey.

1974 CAMPBELL, Capt. the Hon. A. J. C., Holne Rose, Cawdor, Nairn, Scotland.

1977

1950

CAMPBELL, D. N., 16 Regents Park Terrace, London N.W.1.

1977

†CAMPBEL, L, G., 232 Beulah Hill, London S.E.19.

$\ddagger$ CAPDEVIlle, B., Ocean Park Motel, 2690 46th Avenue, San Francisco, Calif. 94116.

1975 CARP, L. W., L'Ermitage, 1171 Bougy-Villiers, Switzerland.

1976

1966

1978

CARSWEll, Prof. J., 3E Carlisle Place, London S.W.1.

CARTER, F. W., Dept. of Geography, University College, Gower Street, London W.C.1.

1959 CASPARIS, J. G. de, Violierenweg, 40, Wassenaar, Holland.

1972 †CATON, P. C. D., 5 Gloucester View, Southsea, Hants.

HON. 1969 CERULLI, H. E. Dr. E., 11 Via Lovanio, Rome.

1968 CHAHIN, M., East Braynes, 27 Church Street, Wiveliscombe, Somerset.

1965 ChaKraborty, Pandit S., Bhagya Ganana Karjaloy, P.O. Ghatal. Dist. Midnapore, West Bengal.

1967 CHAKRAVORTY, N. P., Vill-Nabapally P.O., Natagarh, 24 Parganas, W. Bengal.

1964 †CHALUKEY, S. S. A. P., Kalyani-Kothi, Radha Ganj, Dewas, M.P., India.

1964 CHAN, Dr. L. H. M., 154-164, Argyle Street, Balwin Court, Flat 8-C, Hong Kong.

1967 CHAUDHRY, A. B., P.O. Box 84, Mecca, Saudi Arabia.

1974 CHAWNER, Miss E., Y.W.C.A., 4 New Cantonment Road, Dehra Dun, India.

1976 Chennakesavan, Prof. S., No. 1. 5th Cross Street, C.I.T. Colony, Mijrapore, Madras 600004. 
1959 CHI, Prof. R. S.-Y., East Asian Languages \& Literatures, Goodbody Hall, Indiana University, Bloomington, Indiana 47401. ChIRURG, J. T., Box 4166, Sather Gate, Berkeley, Calif. 94704. CHO, S.-B., Tüvangs-vägen 34, 75245 Uppsala, Sweden.

Choudhuri, Mrs. J. J., c/o Edmonds, Elmfield, New Barn Lane, Cheltenham, Glos.

1960 CHOwDHuRY, Sh. D., c/o The Indian Iron \& Steel Co. Ltd., P.O. Kulti, Dist. Burdwan, West Bengal.

§CHRISTIE, A. H., 14B Elsworthy Terrace, London NW3 3DR.

CLARKE, G. E., Lincoln College, Oxford.

CLAYTON, H., Bolton House, 19-20 Bolton Street, London W.1.

Cleveland, R. L., History Department, University of Regina, Saskatchewan, S4S 0AZ, Canada.

1972

1972

1969

1978

1962

1937

1969

COATES, A., Flat 303, 80 Macdonnell Road, Hong Kong.

COLliN, P. H., 31 Teddington Park, Teddington, Middlesex.

$\$$ CONE, Mrs. M., Jasmine Cottage, Whittonditch, Ramsbury, Wilts.

COOK, M. A., S.O.A.S., Malet Street, London, WC1E 7HP.

CORREIA-AFONSO, Rev. Fr., S.J., St. Xavier's College, Bombay 400001.

COULSON, Mrs. W., O.B.E., 4 Little Boltons, London SW10 9LP.

1968

1960

1970 COX, A. G., 13 Cumberland Road, Kew, Richmond, Surrey.
HON. CAUSA 1978 CRAWFORD, Miss M. D. C., 70 Abingdon Villas, London W.8.

CoUsins, L. S., Dept. of Comparative Religion, University of Manchester, Manchester M13 9P1.

COWAN, Prof. C. D., SOAS, Malet Street, London WCIE 7HP.

COWAN, D., c/o Lloyds Bank Ltd., P.O. box 4, 3 Sidney Street, Cambridge CB2 $5 \mathrm{AQ}$.

1972 CRAWford, Prof. S. C., Dept. of Religion, University of Hawaii, Honolulu 96822.

1972 CRAWSHAW, A. F. B., 92 Talfourd Road, London SE15 2NZ.

1960 †CROFTS, J. N., St. Anne's Cottage, Clifton, Deddington, Oxon.

1970

1973

1973

1972

1975

1976

CROWE, Mrs. Y., 18 Markham Street, London SW3 3NP.

CUlleN, G. F. H., 154 Franks Road, Dovercourt, Essex. CULlEN, Mrs. P. V., 154 Franks Road, Dovercourt, Essex. CULlingS, Mrs. L. D., Langton Hall, Market Harborough, Leics. CUSHMAN, Dr. J., Australian National University, Canberra. CUTHBERT, Miss E., 26 Raleigh Street, London N.1.

DADA BHOY, A. D. P.O. Box 648, Kuala Lumpur.

DALla PICCOLA, Miss R. L., Bergstrasse 152/5, D69, Heidelberg.

versity, P.M.B. 3011, Kano, Nigeria.

1963 DARKE, H. S. G., Faculty of Oriental Studies, Sidgwick Avenue, Cambridge.

1974 DAS, A., 18/2 Chaku Khansama Lane, Calcutta 9.

1973 †DAVIES, Prof. A. M., Somerville College, Oxford.

1960 DAVIES, Prof. D. M., 35 Wells Road, Hindringham, Nr. Fakenham, Norfolk.

1976 DAVIS, M., 15 Ivor Street, London N.W.1.

1921 HON. 1954 DE, Dr. S. K., 19A Chaudhuri Lane, Shyambazar, Calcutta 4.

1959 DEAN, W. K. H., Dept. of English, University of Otago, Box 56, Dunedin.

1965 DEANE, R. J. H., 15 Parkhill Street, Pearce SCT 2607, Australia.

HON. 1954 DENY, Dr. J., Chalet des Glycines, Gérardmer, France.

1948 †DERRETT, Prof. J. D. M., Chiltern House, Lee Common, Great Missenden, Berks.

1976 DESHPANDE, Dr. P. J., Shalya Shalakya Institute of Medical Science, Banaras Hindu University, Varanasi 221005. 
DEVAHUTI, Dr. D., Dept. of History and Political Science, University of Queensland, Brisbane.

HON. 1962 DIA KONOFF, Dr. I. M., Institut Vostokovedeniya, Dvortzovaya Nabareshnaya 18,192041 Leningrad.

1973 DICK, I. W., 58 Millfields, Writtle, Essex.

1974

1976

1976

1954

1977

1974

1961

1970

1973

1968

1954

1971

1974

1971

1970

1954

1939

1969

1976

DICKIE, Dr. J., 69 Nicolson Street, Greenock, Renfrewshire.

DICKSON, M. M., c/o C.B.C., 49/50, Berkeley Street, London W.1.

$\dagger$ \&DIG BY, S. E., 38 Birchington Road, London N.W.6.

DIMES, Miss E. M., 302 Grange Road, London S.E.19.

DINEHART, F. E., 20 Shelley Road, South Meriden, 106450.

DOBBINS, Dr. K. W., P.O. Box 1189, Minnetonka, MN 55343, USA.

DOE, D. B., M.B.E., Beeley Old Hall, Beeley, Matlock, Derbyshire.

DOGHAIM, Prof. M., Faculty of Arts, University of Libya, Benghazi, Libya.

DONNELLY, P. J., St. John's House, Writtle, Essex.

DORMER, Mrs. D., Bowdown House, Newbury, Berks.

§DOWseTT, Prof. C. J. F., Pembroke College, Oxford.

DOWNER, Prof. G. G., University of Leeds, Yorkshire.

DRISKELL, M. B., 21 Harrington Road, St. John's, Worcester.

†DRIVER, J. E. S., 58 Eccleston Square, London S.W.1.

DUdBRIDGE, Dr. G., 4 Coolidge Close, Headington, Oxford OX3 7NP.

$\uparrow \S$ DUNCANSON, D. J., O.B.E., 26 Leinster Mews, London W.2.

DUNLOP, Prof. D. M., 46 Owlstone Road, Cambridge.

§DUNN, Prof. C. J., S.O.A.S., Malet Street, London, WC1E 7HP.

DURRANS, Dr. B., Museum of Mankind, 6 Burlington Gardens, London W1X 2E.

$1946 \uparrow$ DUTT A, Sri D. C., 42 Akhawra Road, P.O. Agartala, Tripura, India.

1967 EAston, Rev. D. A., Tir-Nan-Og, RD2, Box 526, Guildford Schoolhouse Road, New Paltz, N.Y. 12561.

1972 EBIED, Prof. R. Y., Dept. of Semitic Studies, University of Leeds, Leeds LS2 9JT. LS2 9JT.

1930 tEDmONDS, C. J., C.M.G., C.B.E., 5 Long Slip, Langton Green, Nr. Tunbridge Wells, Kent.

1965 EDRIDGE, T. A., Julianstraat 15, Leiderdorp, Leiden, Holland.

1951 EDWARDES, M., Dalchreichart, Glenmoriston, Inverness-shire IN3 6YS.

1976 EILENBERG, Prof. S., 65 Roebuck House, Stag Place, London S.W.1.

1962 EILTS, H. E., H. F., Embassy of the U.S.A., Cairo.

1958 ELFENBEIN, Dr. J., 8 Hamlea Close, London S.E.12.

HON. 1955 ELISSÉEF, Prof. S., 75 Boulevard Pereire, Paris XVIIème.

1965 ELPHICK, O. J., 1 Elsynge Road, London S.W.18.

1963 ELWELL-SUTTON, Prof. L. P., 5 Merchiston Gardens, Edinburgh EH10 5DD.

HON. 1969 EMENEAU, Prof. M. B., Dept. of Linguistics, University of California, Berkeley, Calif. 94720.

1964 †EMMERICK, Dr. R. E., Seminar für Geschichte u. Kultur d. Vorderen Orients, 2 Hamburg 13, Rothenbaumschausee, W. Germany.

HON. 1978 ENOKI, Prof. T., Toyo Bunko (The Oriental Library), Honhamagomo, 2 Chome, 28-31, Bunkyo-Ku, Tokyo.

1965 †ETHELL, B. D., 8 Felden Street, London S.W.6.

1963 EVANS, Mrs. M., Ruskin School of Drawing, 74 High Street, Oxford.

1976 EVELEIGH, M. L., 121 Endlesham Road, London S.W.12.

1973 †EWART, Mrs. S., Flat C, 3 Kensington Park Gardens, London W.11.

1949 †EYermanN, Col. L. M., PO Box 1750, Santa Fe, New Mexico, 87501.

1974 FALK, Dr. M., "Savitri”, Chamonix Mt. Blanc, France 74400

1975 FALK, S. J., 5 Kensington Park Gardens, London W11 3 HB.

1970 FALKINER, R., 15 Yarrell Mansions, Queens Club Gardens, London W.14. 
1965 FARAH, Prof. C. E., 160 Klaeber Court, University of Minnesota, Minneapolis, Minnesota 55455.

FARRINGTON, A. J., 197 Blackfriars Road, London S.E.1.

1979

FASANA, E., via Zanella 44/7, 20133 Milan.

1961

1969

1959

1974

1928

1977

1977

FEHERVARI, G., 58 Woodlands, North Harrow, Middlesex HA2 6EW.

FEISTEL, Dr. H.-O., Schoelerpark 7/IV, D 1000 Berlin 31.

†FERGUSON, Rev. J. P., Craigneish, Albany Road, Douglas, Isle of Man.

FERGUSON, M., 28 Hornton Court, Campden Hill Road, London W.8.

FIELD, H., 3551 Main Highway, Coconut Grove, Miami, Florida 33133.

FEAKIN, P., 2 Vincent Close, Bromley, Kent.

1971

1964

1975

FINN, L., c/o Dr. Dunn, 70 Woodside Avenue, London N.10.

FISCHER, Prof. Dr. K., Helsterbachercott im Kottslefen 10, 533 Konigswinter 41 , Germany.

FISCHER, Prof. C. A., 18A Avenue Road, St. Albans, Herts.

FLETCHER IV, Prof. J. F., Dept. of East Asian Languages, Harvard University, Cambridge, Mass. 02138.

1974

1948

§̧FoRD, J. F., C.M.G., O.B.E., 10 Raymond Road, London SW19 4AP.

1975

1966

1968

1970

1960

1972

1958

1967

1958

†FRANKE, Prof. Dr. H., Universität München, Ostasiatisches Seminar, Munich.

FRANSES, M. D. A., The Textile Gallery, 4 Castellain Road, London W.9.

FREEMAN-GrenVILLE, Dr. G. S. P., North View House, Sheriff Hutton, Yorks.

FREMANTLE, Miss F., 69 Campden Street, London W.8.

FREYTAG, R. A., First National City Bank, PO Box 2221, New York, NY10022.

FRIEDMAN, D. L., 47 Wood Lane, London N.6.

†FRITH, Dr. U., 2 Carver Road, London S.E. 24.

FRYE, Prof. R. N., 546 Widener Library, Cambridge, Mass. 02138.

FRYKenberG, Prof. R. E., Dept. of History, 4134 Humanities, Madison, Wisconsin 53706.

FURBER, Prof. H., 2 Gregory Street, Marblehead, Mass. 01945.

HON. 1969 GABAIN, Prof. Dr. A. von, 8233 Anger, Haus Sonnleiten, Germany.

1971 GAHLIN, S., Quiet House, 13 Macaulay Buildings, Bath BA2 6AT.

1970 Galbraith, W. C., c/o Royal Bank of Scotland, 36 St. Andrew's Square, Edinburgh.

1960 TGalviN, Dato J., P.M.N., 63 Fitzwilliam Square, Dublin.

1949 TANDA SINGH, Lower Mall, Patiala 3, Punjab, India.

1971 TGARDINER, Rev. J., 17 Rossie Place, Edinburgh EH7 5SD.

1977 GARDNER, A. N. A., 487 Fulham Palace Road, London S.W.6.

1958

1979

1979

1963

1973

1977

1977

1974

1944

1962

1977

GARDNER, K. B., 1 Duncombe Road, Bengeo, Hertford, Herts SG14 3BY.

$\ddagger$ GARICK, P. A., Tara, 55 Woodham Waye, Woking, Surrey.

GARRIGUES, S., 'Atenisi Institute, PO Box 90, Wuku 'Alofa, Tonga.

GAUR, Dr. (Mrs.) A., 4 Kingswood Close, Surbiton, Surrey.

†GAUR, Dr. R. C., 3/80, Marris Road, Aligarh U.P., India.

GATSIE, Miss C., 3336 Brownsville Road, Pittsburgh, Pa. 15227.

‡GEAKE, Miss J. E., c/o National Westminster Bank Ltd, Old Bank, Bath.

GELBLUM, T., 37 Cascade Avenue, London N.10.

GERSHEVITCH, I., 54 Owlstone Road, Cambridge.

GHOSH, O. K., Flat 4, 5 Russell Street, Calcutta 700016.

GHOUSHBEIGYU, N., 3005, Massachusetts Avenue N.W., Washington D.C. 20008.

1971 GignOUX, Prof. P., 5 Allée du Maconnais, Ferolles-Atilly, 77330 Ozoir-laFerrière, France.

1958 GILBERT-BENTLEY, F. G., 95 The Street, Shalford, Guildford, Surrey. 
1965 GoKHALE, Dr. B. G., Box 7547, Reynolda Station, Winston-Salem, North Carolina.

1973 GoldMAN, P., 28 Davies Street, London W.1.

1967 GOlDWATER, Dr. C., c/o British Museum, Box 1690, London WC1 6XX.

1971 †GOMBRICH, Dr. R. F., 6 Portland Road, Oxford OX2 7EY.

HON. 1954 GONDA, Prof. Dr. J., Van Hogendorpstraat 13, Utrecht, Holland.

1973 §GOODWIN, F. G., 29 Chalcot Square, London NW1 YA8,

1960 †OPA L, Dr. L., 184 Shahrarabagh, Allahabad 3, India.

HON. 1975 GoRDION, Prof. C. H., 130 Dean Road, Brookline, Mass. 02146.

1976 GouDIE, D. J., 123 Ferry Road, P.O. Box 203, Glebe., N.S.W., Australia.

1972 GowING, Dr. P. G., Dansalan Research Centre, P.O. Box 5430, Iligan City, 8801, Philippines.

1978 GRAZIA, B. C. de, 10 York Terrace East, London NW1 4PT.

1977 GREEN, M., Apt 102 Fichtenweg 18, D-7400, Tübingen, W. Germany.

1963 †GREENE, D. B., 12A Woodgrange Mansions, Woodgrange Avenue, Harrow, Middlesex.

1965 GreENFIELD, Prof. J. C., Dept. of Ancient Semitic Languages, Hebrew University, Jerusalem.

1961

1976

†GRENFELD, A. J., 4785 New Salem Road, RD2, Voorheesville, N.Y. 12186.

1976

1976

1970

1957

1958

1976

1967

1971

1956

1968

1961

1973

1974

GROOM, N. St. J., 24 Sheffield Terrace, London W8 7NA.

GROOT, Dr. A. H. de, Vakgroep Arabisch, Perzisch, Turkisch en Zuid Semietisch, Rapenburg 61, Leiden, Holland.

GrosliER, Prof. B. P., Directeur de Recherches, C.N.R.S., 13 Square Gabriel Fauré, Paris 78117.

GRUBE, E. J., Villa van Axel, Altivole, Treviso, Italy.

GUlBE KIAN, E. V., 10 Graham Avenue, Mitcham, Surrey.

†GuPT A, Prof. P. C., 125 Rashabikari Avenue, Calcutta 700029.

GUPTA, P. L., J12/15 Bowlia Bagh, Ramkatora, Varanasi 221002.

GUPTA, R. D., S.O.A.S., Malet Street, London WC1E 7HP.

GUPTA, S. P., C-23 Ditakusha Colony, Lucknow 2.

†GUREWICZ, S. B., 31 Blandford Avenue, Waverley, Sidney, Australia.

GURU, Dr. L.S. V., Dept. of Basic Principles, Institute of Medical Sciences, Benares Hinđu University, Varanasi 5.

1965

1974

1953

1978

1959

1978

1974

1972

HAELlQUiST, Dr. K.-R., Kung Oscars Vag 3, 22240 Lund, Sweden.

†HAIG, R. W., present address unknown.

HAIM ENDORF, Prof. C. von Fürer-, S.O.A.S., Malet Street, London WC1E 7HP.

HALASI-KUN, Prof. T., 114 Morningside Drive, New York, N.Y. 10027.

HALDANE, Dr. J. D., 221 Felsham Road, London S.W.15.

HALL, Prof. D. G. E., 4 Chiltern Road, Hitchen, Herts.

HALPERIN, Dr., c/o Coudert Brothers, 31st Floor, Alexandra House, 20 Chater Road, Hong Kong.

Hambly, Mrs. M., 72 Dessa Drive, Hamden, Connecticut 06517.

1973 HANAWAY, W. L., Jr., Dept. of Oriental Studies, University of Pennsylvania, Philadelphia, Pa. 19174.

Hon. CAUSA 1975 HANSMAN, Dr. J., c/o S.O.A.S., Malet Street, London WC1E 7HP.

1971 HARDIE, P., 19 Canynge Square, Bristol BS8 3LA.

1974 HARDY, F., 2 Denmark Road, Reading, Berks.

1958 HARDY, P., 9 Longcroft Road, Edgware, Middlesex.

1972 HARE, R. E. O., present address unknown.

1962 \$HARLE, J. C., Dept. of Eastern Art, Ashmolean Museum, Oxford.

1972

HARROW, L., 32 Melford Road, London E.11. 
†HART, G., Dept. of Classics, 38 North Bailey, Durham.

HART, G. A. J., 15 Merryfields Avenue, Hockley, Essex.

HASAN, Sheikh K., 9-D Officers' Colony, Garden Road, Karachi 3, Pakistan.

$\ddagger$ HASSAN, A. H., Eliot College, University of Kent, Canterbury, Kent.

HASTIE, J., 74 Montholme Road, London S.W.11.

HatTEM, W. C. van, CP.188, Rua Gen. Carneiro 215, Ponta Grossa 84100, Parana, Brazil.

HATTO, Prof. A. T., Arrandine Cottage, Wise Lane, London, N.W.7.

HATtON, A. G., Ipswich Museums \& Art Galleries, The Museum, High Street, Ipswich IP1 3QA.

$\dagger \S$ HAY, M. C., Perlis, Burpham, Arundel, Sussex.

HAYES, J. W., New Territories Administration, Tsuen Wan, Hong Kong.

HAYES, P. A., 53 Wetherby Mansions, Earls Court Square, London S.W.5.

\$HAYLEY, Miss A., $1 \mathrm{~A}$ Allison Grove, London SE2 7ER.

HAYWARD, J. D., Windles, 63 Violet Lane, Croydon CR0 4HD.

†HAYWARD, W., 7459 Restful Avenue, Orlando, Florida 32807.

HAYWOOD, J. A., Flat 4, The Rowans, Prince Edward Road, Lewes, Sussex.

HEAD, R. V., 20 Homefield Road, London S.W.19.

HEALEY, J. F., Dept. of Semitic Languages, University College, Cardiff CF 11 XA.

HEATHCOTE, T. A., Officers Mess, R.M.A., Sandhurst, Camberley, Surrey GU15 4PQ.

HEDLEY, P., 25 Batchelor Street, London N.1.

$\dagger$ HEIN, Prof. N. J., 409 Prospect Street, New Haven Conn. 06510.

§HENDERSON, Prof. E. J. A., S.O.A.S., Malet Street, London WC1E 7HP.

HENNING, Major D. E. H., 4 Kingswood Close, Surbiton, Surrey.

HER BERT, Miss P., British Library, Dept. of Oriental Manuscripts, Great Russell Street, London W.C.1.

$\dagger$ HERCUS, Mrs. L. A., A ustralian National University, P.O. Box 4, Canberra.

HERviEU, A. G., La Placette, F06570 St. Paul-de-Vence, nr. Nice, France.

1978 HettiaratchI, Prof. D. E., 46 Sir Marcus Fernando Mawatha, Colombo 7, Sri Lanka.

HEYWOOD, C. J., S.O.A.S., Malet Street, London WC1E 7HP.

HIGGS, A. S., Officers Mess, 1st Bn., Royal Welch Fusiliers, BFPO 41.

HILDITCH, K. R., 32 Butts Road, Penn, Wolverhampton, Staffs.

HILL, D., 18 Holly. Hill, Lond on N.W.3.

HILL, Dr. D. R., 3 Amey Drive, Great Bookham, Surrey.

HILMI, Dr. P. R., 7 Ferry Road, London S.W.13.

HINDS, G. M., Faculty of Oriental Studies, Sidgwick Avenue, Cambridge.

HINNELLS, J. R., 10 St. Brannock's Road, Chorlton-cum-Hardy, Manchester 21.

$\dagger$ HINÜ BER, Dr. O. von, Seminar für Indologie, Postfach 3980, 65 Mainz, Germany.

HISKETT, M., S.O.A.S., Malet Street, London WC1E 7HP.

\$HITSON, Miss J. A., 23 Christchurch Hill, London N.W.3.

HOARE, O., 6 Tregunter Road, London S.W.10.

HOBART, P. M., 20 Sheffield Terrace, London W.8.

HOBHOUSE, N. A., 1/126 Hamilton Terrace, London N.W.8.

HOBOHM, M. A. H., 39 Montague Square, London W.1.

HODGKIN, H., 12 Addison Gardens, London W.14.

HofFMAN, F. J., Education Center, Camp Zama-shi, Kanayawa-ku, Japan 228.

HOLKAR, Prince Richard, Ahilya Fort, Maheshwar, Nimar, M.P., India.

HOLMES, R. E., 12 St. Andrews Drive, Ilkeston, Derbyshire.

HOLT, Prof. P. M., North View, South Heath, Gt. Missenden, Bucks.

HONEY, P. J., Baddesley House, 43 Royston Park Road, Hatch End, Mx.

†HONEYman, Prof. A. M., Old town of Ardgay, Ardgay, Ross, Scotland.

†HONY, H. C., Hyde Cross, Marlborough, Wilts.

HOOK, B., Dept. of Chinese, The University, Leeds, Yorkshire.

HOOKER, M. B., Eliot College, University of Kent, Canterbury. 
HOPGOOD, J. B. C., 3 Bach Lane, The Street, Ramsey, Harwich, Essex. HORNER, Miss I. B., 62 South Lodge, Circus Road, London N.W.8. HOWAY-FrANKLIN, Mrs. T., Mariposa, The Avenue, Ascot, Berkshire. HOWARD, Rev. S., The Vicarage, Eggleston, Barnard Castle, Co. Durham DL12 OAN.

HUDSON, Major E. R. B., PO Box 1146, Chiang Mai, Thailand.

HUMBACH, Prof. H., Postfach 3980, D65 Mainz, Germany.

Huntingdon, Prof. J. C., Division of Art \& History, Ohio State University, Columbus, Ohio 43210.

HUSSAIN, A. B. M., University of Rajshahi, Dept. of Islamic History \& Culture, Rajshahi, Bangladesh.

HUSSAIN, Dr. S. A., 63 Angappa Naick Street, Madras 600001.

HUTT, A. M., 13 Letchford Gardens, London N.W.10.

HUYLER, S. P., 46 Collingwood Avenue, London N.10.

HON. 1978 INALCIK, Prof. H., 1126 East 59th Street, Chicago, Illinois 60637.

1965 INSLER, Prof. S., 284 Orange Street, New Haven, Conn., 06510.

1970

1966

1976

ION, Mrs. H., Max-Planck-Str. 61A, 6905 Schriesheim, West Germany.

§IRVINE, Dr. A. K., 21 Staples Road, Loughton, Essex.

1946

IRVINE, Mrs. V. E., 21 Staples Road, Loughton, Essex.

1970 $\dagger$ IRWIN, J., Ashford Chace, Steep, Petersfield, Hants GU32 1AB.

1960

IRWIN, R., 39 Harleyford Road, London S.E.11.

ISAACS, Dr. H. D., 19 Hall Drive, Mottram-in-Longdendale, Hyde, Cheshire.

1971 JACHIMOWICZ, Dr. E., Gatterburggasse 15/5, A-1190, Vienna.

1955 JACOB, Mrs. J. M., Angkor, 1 Woodham Way, Woking, Surrey.

1976 JACQUES, Prof. C., Ecole Pratique des Hautes Etudes, 45 rue des Ecoles, Paris 75006 .

1972 JAEghER, Rev. R. J. de, 86 Riverside Drive, New York, N.Y. 10024.

1978 JAGGI, Dr. O. P., Vallabhbhai Patel Chest Institute, University of Delhi, Delhi 11007.

1968 JAHN, Prof. K., Müllnergasse C/31, 7090 Vienna.

1967 JAIRAZBHOY, N. A., Dept. of Music, U.C.L.A., 405 Hilgard Street, Los Angeles, Calif. 90024.

HON. 1971 JARRING, H. E. Dr. G., Karlavägan 85, 11459 Stockholm.

1971 JELLICOE, Countess, 20 Chapel Street, London S.W.1.

$1971 \dagger$ JHA, Prof. J. C., opposite the Main Gate of the Rajendranagar Stadium, Patna 16, Bihar State, India.

1952 Johns, Prof. A. H., Australian National University, P.O. Box 4, Canberra.

1960 JOHNSON, Miss D. M., 60 Vivian Avenue, Wembley, Middlesex.

1960 JOHNSTONE, Prof. T. M., S.O.A.S., Malet Street, London WC1E 7HP.

1964 JONES, A., St. Cross College, Oxford.

1974 JONES, Mrs. D. L., Priory Cottage, 102 St. Paul's Road, London N.1.

1976 JONES, M. D. L., 130 Kenilworth Court, Lower Richmond Road, London S.W.15.

1959 JONES, R., S.O.A.S., Malet Street, London WC1E 7HP.

1965 JUNANKAR, N. S., 25 Welldon Crescent, Harrow, Middlesex HA1 1QP. 
1966 KAIL, Dr. O. C., Pushpar Vihar 1, 159 Colaba Road, Bombay 5.

1977 KAPUR, R. A., Merton College, Oxford.

1972 KARPAT, Prof. K.H., 3211 Humanities Building, University of Wisconsin, Madison, Wisconsin 53706.

1970 \#KASABOVA, Mrs. S., 23 Stratford Villas, London N.W.1.

1966 KAYE, Dr. A. S., Dept. of Linguistics, Cal. State University, Fullerton, Ca. 92634.

1976

1971

1971

1950

$\mathrm{KEACH}, \mathrm{K}$. L., 2501 Canterbury East 316, Seattle, Washington 98102.

KEELER, P., 29 Campion Road, London S.W.15.

KENNEDY, R. E., 7063 Lanewood Avenue, Apt. 106, Hollywood, Calif. 90028.

$\nmid$ KENSDALE, W. E. N., c/o FCO, (Algiers), King Charles Street, London SW1A $2 A H$.

1969 KESSLER, Dr. C., 5 Winchmore Drive, Trumpington, Cambridge CB2 2LW.

1966 KHALEEQ, Dr. A. S., Government Islamia Sec. School, Toba Tek Singh, Dist. Lyallpur, Pakistan.

1972 KHAN, Prof. B. R., 59 Ravenslea Road, London SW12 8SL.

1979 KHAN, I., Director of Archaeology, Government of Pakistan, Karachi.

1977

1959

1959

1934

1972

1968

1978

1976

1974

1967

1978

HON.

1978

1973

1965

1979

1965

1972

KHAN, M. A. A., Islamic History Dept., Rajshahi University, Bangladesh.

KHAN, Dr. M. S., Flat 304, Wellesley Mansions, 443 Wellesley Street, Calcutta 16. $\dagger$ KHAN, S., W.P.E.S. 11, V.P.O. Nawan Shahr, Hazara, Pakistan.

KHANNA, Dr. K. C., Ravensdale, Simla 2.

KHOBRE KAR, V. G., Elphinstone College, Bombay 1.

$\dagger$ KHOROCHE, P. A., 4 Lewes Crescent, Brighton, Sussex.

KIEFFER, Dr. C. M., 2 rue Steinbach, 68700 Cernay, France.

KINANY, Dr. A. K., 39 Ave René Coty, 75014 Paris.

KING, G. R. D., c/o Mrs. G. King, 14 Rosemary Close, Roderick Avenue, Peacehaven, Sussex.

KNAPPERT, J., 2 St. Andrews Avenue, Harpenden, Herts.

KNOX, J. R., Dept. of Oriental Antiquities, British Museum, London WC1 3DG.

1957 KONONOV, Prof. A. N., Ul. Golgoya 4/1, KV46, Leningrad 191065 D-65.

KOPEINIG, W. F., 5701 25th N.E., Seattle, WA 98105.

KOSSOW, R. E., 3 Headfort Place, London S.W.1.

$\dagger$ KOTHARI, H. R., 12 India Exchange Place, Calcutta 1.

KRAT Z, E. V., S.O.A.S., Malet Street, London WC1E 7HP.

KREMER, Mrs. A. B., 95a Belsize Lane, London N.W.3.

1974

KREYENBROEK, Dr. G., Cornelius Krusemanstraat 21 II, Amsterdam.

Kentucky 42071.
KuMA,

F.E.F. 1975 KÜNERALP, H. E., Z., Embassy of Turkey, Madrid.

1965 KUNST, Dr. A. V., 85 York Mansions, Prince of Wales Drive, London S.W.11.

1977 KUNWAR, R. K., 164 Lai Basti, Aclapur, Allahabad, India.

1959 KURIAN, G., Dept. of Sociology \& Anthropology, University of Calgary, Calgary.

1974 KURISU, M., 12 Uenomiya, Tennoji, Osaka, Japan.

1976 LAFOnT, Prof. P. B., Ecole Pratique des Hautes Etudes, 45 Rue des Ecoles, 75006 Paris.

1945 LAMBTON, Prof. A. K. S., O.B.E., Gregory Kirknewton, Wooler, Northumberland.

1947 HON. 1963 LAMOTTE, Rev. Prof. E., Place du Roi Vainqueur 15, B.P.10, B-1040, Brussels.

1972 §LANCASTER, Miss J. C., C.B.E., 43 Craignair Road, London SW2 2DQ.

$1949+\S L A N G$, Prof. D. M., S.O.A.S., Malet Street, London WC1E 7HP.

1968 LANGE, H. P., c/o Project Hope, 42 Sharia El-Batal Ahmed Abdel Aziz, Madinetel-Mohandisee, Dokki, Cairo.

1977 LARIVIERE, R., 820 Williams Hall, C.U., Philadelphia 19174.

1963 LATHAM, J. D., Dept. of Near Eastern Studies, The University, Manchester 13. 
1963

1977

1970

1968

1978

1977

1965

(1965)

HON 1978 LEWICKI, CinC D. T. 30069 KokO

I, Prof. Dr. T., 30-069 Krakow, UI Warmiska 5, Poland.

1978

1939

1967

1977

1968

HON. 19

1965

1964

1926

1974

1959

1957

1959

1952

1971

1976

1946

1968

1965

1972

1964

1977

1966

1935

1973

1971

1968

1979

1946

1958

1973

§LATtimore, Prof. O., 26 Rue de Picpus, 75012 Paris.

LAWSON, S. D., 49 Stratford Street, Oxford OX4 1SP.

$\ddagger$ LAXMAN, Mrs. S., 11 Queens Grove, London N.W.8.

LEACH, J., 7 Felden Street, London S.W.6. N.Y. 11724

LEE, W. C. -C., 42, Lord Sterling Drive, Parsippany, N.J. 07054, U.S.A. Avenue, Cincinnati, Ohio 45220.

LEWTHWAITE, Mrs. R., 14 Edwardes Square, London W8 6HE.

$\S L E W I S$, Prof. B., Institute for Advanced Study, Princeton, New Jersey 08540.

LEWIS, Dr. G. L., St. Anthony's College, Oxford. University, New Brunswick, N.J. 08903.

LIEBER, Dr. A. E., Rehove Balfour 13, Jerusalem.

1967 LIGETI, Prof. L., Belgrad, rkp. 26, 1056 Budapest, Hungary.

LIGHT BOWN, R. W., 27 Chalcot Crescent, London N.W.1.

$\dagger$ LIN, U San, 299 Bo Aung Gyaw Street, Rangoon.

LINDE, C. de, The British Institute, 11 Rue de Constantine, 75002 Paris.

§̧LINDGREN, Dr. E. J., Sunbourn, Harston, Cambridge. M13 9PL.

§LINGS, Dr. M., 3 French Street, Westerham, Kent TN16 1 PN. National University, Canberra. CB3 9DA.

$\dagger$ LOHUIZEN de LEEUW, Prof. Dr. J. E. van, van Breestraat 22, Amsterdam.

LOMAN, D. E. F., 12 Suffolk Road, London S.W.13.

LOMBARD, Prof. D., 52 rue Blomet, 75015 Paris. Islands, British West Indies. Canberra. Washington, Seattle, Washington 98105. Books, Great Russell Street, London WC1B 3DG.

§LOWICK, N. M., 5 Teignmouth Lodge, Teignmouth Road, London N.W.2.

LOWLE, D. A., 13 Norwich Road, Liverpool L15 9HL.

LOWRY, J., Indian Section, Victoria \& Albert Museum, London S.W.7. C.I.

LUFT, Dr. P., German Historical Institute, 42 Russell Square, London W.C.1. 3074, Frieze Buildings, Ann Arbor, Michigan 48104.

LYLE, M. G., c/o B/M/MG Lyle, London W.C.1.

LYSTER-BINNS, N., 61 Catherine Place, London SW1E 6HB. Dorset.

†MACDOWALL, D. W., Admont, Dancer's End, Tring, Herts HP23 6HY.

LEE, Mrs. M., PO Box 112, Harbour Road, Cold Spring Harbour, Long Island,

LEHMAN, Dr. O.E., Curator's Office, Hebrew Union College, 3101 Clifton

LI, Dr. P., Dept. of Foreign Languages \& Literature, Livingstone College, Rutgers

LING, Prof. T., Dept. of Comparative Religion, The University, Manchester

LIU, Prof. T. Y., Dept. of Chinese, Faculty of Oriental Studies, Australian

$\S L O E W E$, Dr. M. A. N., Faculty of Oriental Studies, Sidgwick Avenue, Cambridge

LONG, A.C. E., C.M.G., C.B.E., Box 131, Savannah, Grand Canyon, Cayman

LOOFS, Dr. H. H. E., Faculty of Oriental Studies, Australian National University,

LORAINE, M. B., Dept. of Near Eastern Languages, Denry Hall, Univ. of

LOSTY, J.P., The British Library, Dept. of Oriental Manuscripts \& Printed

†LUCE, Prof. G. H., C.B.E., Uplands, Grande Route, St. Lawrence, Jersey,

LUTHER, Prof. K. A., University of Michigan, Dept. of Near Eastern Studies,

MACDERMOT, B. C., C.B.E., M.V.O., The Old Rectory, St. James, Shaftesbury,

MACHAdo MOURET, Prof. Dr. O., Calle Jorge, Newberry 2530-2-1, Buenos Aires. 
1973 Mackintosh, D. J. H., Dept. of Extra Mural Studies, University of Hong Kong, Hong Kong.

1943 TMACLAURIN, E. C. B., 53 Langley, 12 Prospect Avenue, Cremorne, N.S.W. 2090, Australia.

1973 MAGgS, H. C., 11 Oatlands Drive, Weybridge, Surrey.

1965 MAJUmdar, Dr. A., 90/8 Connaught Circus, New Delhi.

HON. 1957 MAJUMDAR, R. C., 4 Bapin Pal Road, Calcutta 26.

1951 MAKDISI, Prof. G., 841 Williams Hall, University of Pennsylvania, Philadelphia, Pa. 19174.

1959 MAKOWIECKA, Mrs. J., 2 Amherst Road, London W.13.

1977 MALliSON, Mme. F., Les Mentizs, 30170 St. Hyppolyte-du-Fort, France.

1976 MARKS, Dr. J. A., 512 Allerton Road, Liverpool L18 9UY.

1976 MARKS, Mrs. V. C., 512 Allerton Road, Liverpool L18 9UY.

1960

1973

1977

1968

1974

1978

1970

†MARRISON, Rev. Canon, G. E., 85 Warwick Road, Thornton Heath, Surrey.

MARSHALL, Prof. D. N., University of Bombay, Bombay 32.

MARSHALL, Miss H., S.O.A.S., Malet Street, London WC1E 7HP.

$\dagger$ \$MARShall, P. S., Third Floor, 25 Queen Anne's Gate, London S.W.1.

MARTELL, W. H., La Trobe University, Bundoora, Victoria 3083, Australia.

1974

1975

1969

1954

1972

1972

1972

1977

1966

1974

1974

1954

1975

1965

MARTIN, Prof. B. G., Ballantyre Hall, Bloomington, Indiana 47401.

MASTERSON, Miss M., 355 Fair Oaks Street, San Francisco, Calif. 94110.

MATTHEWS, D. J., S.O.A.S., Malet Street, London WC1E 7HP.

MATTHEWS, S. R., 44 Northolme Road, London N.5.

MAURER, Prof. W. H., 2333 Kapiolani Blvd., No. 813, Honolulu, Hawaii 96826.

MAY, Dr. J., 68 Avenue de Rumine, CH 1005, Lausanne, Switzerland.

MCCHESNEY, R. D., Dept. of Near Eastern Languages and Literature, New York University, 50 Washington Square So., New York N.Y. 10003.

MCDOUGALL, Miss N. K., 9 Hill Street, Innaloo, Western Australia.

MCEWEN, E., 10 Richmond Way, London E.11.

MCFARLAND, J. P., 10 Fulwood Park, Liverpool L17 5AM.

MCGREGOR, R. S., 79 Thornton Road, Cambridge.

MCQUIRK, R. H., 202c Hammersmith Grove, London W.6.

MCQUIR K, Mrs. S. N., 202c Hammersmith Grove, London W.6.

MCKANE, Rev. W., 51 Irvine Crescent, St. Andrews, Scotland.

MCKINNON, E. E., 120 Uris Hall, Cornell University, Ithaca, New York 14853.

MCLEOD, Dr. W. H., Dept. of History, University of Otago, PO Box 56, Dunedin, New Zealand.

$1924 \dagger$ MCMillen, Prof. O. W. 510 N. Brookside Drive, Little Rock, Arkansas 72205.

1976 MEARNS, Mrs. L., Dept. of Anthropology, University of Adelaide, GPO Box 498, Adelaide 5001.

1960 MEDLEY, Miss M., Percival David Foundation, 53 Gordon Square, London W.C.1.

1970 MEGALLY, Dr. F., 509 Duncan House, Dolphin Square, London S.W.1.

1972 Melamparampil, Rev. Dr. J., Melamparampil House, Podiyadi P.O. via Tiruvalla, Kerala, India.

1973 MELlOTT, R. L., Fogg Museum of Art, Harvard University, Cambridge, Mass. 02138.

1962 §MÉNAGE, Prof. V. L., 3 Tite Street, London S.W.3.

1949 MEREDith-OWENS, Prof. G. M., Dept. of Islamic Studies, University of Toronto, Toronto 5.

1972 MERRIMAN, Dr. B. M., 85 Holland Park, London W.11.

F.E.F. 1971 MIKASA of Japan, H.I.H. Prince, 2-1-6 Moto-Akasaka, Minato-Ken, Tokyo.

1945 Mills, Dr. J. V., 90 Avenue de Sully, 1814, La Tour de Peilz, Vaud, Switzerland.

1965

1974

$\dagger$ MILNE, A. C. C., c/o Midland Bank Ltd., 102 High Street, Lymington, Hants.

1978

MILNER, A., Keynes College, The University, Canterbury, Kent.

1977

MISHRA, Dr. S. M., 339 Trivaniganj, Naubasta, Lucknow.

MITCHELL, T. C., 3, Dept. of Western Asiatic Antiquities, British Museum, Great Russell Street, London W.C.1. 
MitChELL, V. J., c/o 25 Turner Street, Lambton, New South Wales 2299.

MITCHINER, J. E., 195/1 Park Street, Calcutta 700017.

MITCHINER, M. B., "Ravenhill", Rectory Park, Sanderstead, Croydon, Surrey CR2 9JR.

MONCK, Hon. Mrs., Aldern Bridge House, Newbury, Berks.

1968 00920.

MORGANS, D. A., Bleakholt Animal Sanctuary, Bury Old Road, Shuttleworth, Ramsbottom, Lancs. BL0 0RX.

1968 MORTON, A. H. Mulberry House, Little Wilbraham, Cambridge.

MORTON-SMITH, R., Dept. of Sanskrit \& Indian Studies, University of Toronto, Toronto 181 .

MOTTAHEDEH, R. Y., 225 Fifth Avenue, New York, N.Y. 10010.

MUKHERJEE, B. N., 36 Jatin Das Rd., Calcutta 700029.

MUKHER JEE, T. P., S.O.A.S., Malet Street, London WC1E 7HP.

MUNRO-HAY, S., 33 Rock Park, Rock Ferry, Merseyside.

MURThI, Prof. S. K., Room No. 303, Frontier Hotel, Queen's Road, Delhi-6.

MURTHY, Prof. K. R. S., Dept. of Postgraduate Studies, Government College of

Indian Medicine, Bangalore.
MUTTU CUMARI SWAMY, C.S. V., 4 Thurleigh Court, Nightingale Lane, London SW12 8AP.

NAGAR, J., 129 Attarsuiya, Allahabad 211003, India.

$\dagger$ NAHA RSINGHI, Lt. Maharaj S.S. Sahib Bahadur, of Choota, Udepur, Rewa Kantha District, Bombay.

1973 NAKAHARA, Miss M., International Division, Waseda University, 1-647 Totsukamachi, Shinjuku-ku, Tokyo 160.

HON. 1978 NaKAMURA, Professor H., 4-37-10, Kugayama, Suginami-ken, Tokyo.

1977 NARUMIT, S., 37, Somerton Avenue, Richmond, Surrey TW9 4QF.

1975 NASIA, Mrs. P. T., National Museum of Pakistan, Karachi.

1955 †AYLOR, E. C., P.O. Box 313, Belleville, Ontario, Canada.

1949

1979

1968

HON. 1958 NILAKANT A SASTRI, Prof. K. A., Nileshvar, Edward Elliott's Road, Mylapore, Madras.

1972 NiSBET, J. D. F., P.O. Box 655, Tripoli, Libya.

1954 NORMAN, K. R., 6 Huttles Green, Shepreth, Royston, Herts.

1977 O'BRIEN, A. G., Hareshawmuir, Blackheath, Nr. Guildford, Surrey. Iraq. 
1971 PAGE-BARTON, Mrs. R., 13 Kensington Park Gardens, London W.11. PAN, Dr. S. C. Y., 924 West End Avenue, New York N.Y. 10025.

PANAYOTEAS, S., 30, Iackovidou, Patissa, Athens, Greece 905.

1976

1969

1969

1928

1955

PANDEY, Dr. G., 31 Kherapati Colony, Gwalior 474002, M.P., India.

PANDEY, S. M., 24 Midmore Road, London S.W.19.

PANIGRAHI, Dr. N. K., 1 Urquhart Square, Calcutta 6.

$\dagger$ PARPIA, Y. R., 277 Latymer Court, Hammersmith Road, London W.6.

†PARR, P. J., C/o National \& Grindlay's Bank, 13 St. James's Square, London S.W.1.

1970 PARRY, J.P., 42 Lisburne Road, London N.W.3.

1978 PATTON, D., 545 W. 111 th Street, Apt 5-L, New York N.Y. 10025.

1969 PAVIER, B. H., 24 Freshfield Place, Brighton, Sussex.

1946

1972

1960

1950

HON.

1969

1965

PAYNE, E. M. F., O.B.E., 7 High Street, Barkway, Royston, Herts SG8 8EA.

PAYNE, G. K., 34 Ealing Village, Hanger Lane, London W.5.

PE, Prof. Hla, 25 Pentley Park, Welwyn Garden City, Herts.

§PEARSON, Prof. J. D., 60 Woodstock Road, Nr. St. Alban's, Herts.

1974

1978

F.E.F. 1979 H. E. PHAN WANAMATHEE, Royal Thai Embassy, 30 Queen's Gate, London S.W.7.

1957

1976

\$PHILIPS, Prof. Sir Cyril, S.O.A.S., Malet Street, London WC1E 7HP.

PETERS, H. G. W., 4 Palace Court, London W.2.

PETERSON, B. J., Karlaplan H 10-1, Stockholm S-11522.

PETRUCCIOLI, A., via Goiran 23,00195 Rome.

PEYTON, Wm., Cockhaise, Windfield, Sussex RH16 2QP.

1976

PHILliPS, J. G., c/o Mrs. E. M. Burr, 90 Johnson Street, Tamworth, N.S.W. 2340, Australia.

1978

PIATAGORSKY, A., 46 Caterham Road, London S.E.13.

1976

1976

1958

1969

1975

1956

1975

1969

1964

1969

PICKEN, Rev. Prof. S. D. B., International Christian University, Mitaka, Tokyo.

PINGREE, Rev. D., Box 1900, Brown University, Rhode Island, 02910 USA.

$\$$ PIERONI, N. B., The Polytechnic, Hope Street, Liverpool L1 9EB.

§PINDER-WILsON, R. H., Institute of Afghan Studies, PO Box 3052, Kabul, Afghanistan.

†PINE, L. G., Hall Lodge Cottage, Brettenham, nr. Ipswich, Suffolk.

PINNER, R., 34 Pope's Grove, Twickenham, Middlesex.

POCOCK, Prof. D.F., The School of African \& Asian Studies, University of Sussex, Falmer, Brighton, Sussex.

POLLOCK, M., 40 Carysfort Road, London N16 9AL.

POT AMANIOS-PHILON, Mrs. H., 53 Campden Hill Road, London W.8.

POUR-RESA, M. M., present address unknown.

POWELL, Miss A., University of Technology, Loughborough, Leics.

PRASAD, Dr. G., SS Hospital, Narampur, Mirzapur, U.P., India.

1975

1979

1954

1970

1975

PRESENCER, Dr. A., 92 Greenfield Avenue, Carpenders Park, Oxhey, Herts.

PRIEST, J., Flat 5, Cranleigh Court, 4/5 Leinster Gardens, London W.2.

PRITSAK, Prof. Dr. O., 14 Linwood Road, Wellesley, Mass. 02181.

$\$$ PROCHAZKA, Dr. T., c/o Mr. A. Gibson, 1 Linnet Close, Basingstoke, Hants.

$\uparrow$ PUZPOSAPUTRO, M. S., 1A Exeter Road, London NW2 4SJ.

1963 TQADIRI, K. H., 27 Nant Road, London N.W.2.

1960

1976

1976

1925

1976

1929

1975

QADRI, S. Z. H., Block 8/H 25, Khanewal, District Multan, Pakistan.

QUESNE-BIRD, N. du, Kingston Lodge, Woolley Street, Bradford-on-Avon, Wilts.

RADICE, C. W., 14 Clifton Avenue, Belmont, Surrey.

RAHDER, Prof. Dr. J., 94 Vineyard Road, Hamden, Conn. 06517.

$\dagger$ RAILSBACK, G. H., via Archimede 120,00197 Rome.

$\dagger$ RAM, P., 260/5 Thaparnagar, Meerut City, U.P., India.

RAMACHANDRAN, N., Yusef Building, Seremban, Malaysia. 
RAMESAN, Dr. N., Board of Revenue, Nampally Station Road, Hyderabad 1.

RANGANATHAN, B., Dept. of Ancient History \& Archaeology, The University, Madras 5.

$\dagger$ RAO, Dr. M. S., present address unknown.

RAO, U. R., M.B.E., 1 Ganapathy Colony, Madras 600086.

RASTEGAR, Dr. G. F., Faculty of Letters, Ferdousi University, Meshed, Iran.

RASTOGI, Dr. N. P. R., 215/31 Subhash Marg, Lucknow.

$\dagger$ RAU, Prof. Dr. W., 3553 Reddehausen, Hohlweg 3, W. Germany.

RAWLINSON, Prof. J., Dept. of History, Hofstra University, Hempstead, Long Island N.Y. 11550.

READE, J. E., 12 Museum Mansions, 63A Great Russell Street, London W.C.1.

RESCHER, Prof. N., University of Pittsburgh, Pittsburgh 13.

REYNOLDS, C. H. B., Little Squerryes, Westerham, Kent.

RHALLY, Miss M. C., 6 Eglon Mews, London N.W.1.

RICE, Mrs. F., 23 Ennismore Gardens, London S.W.7.

$\dagger \S$ RICHARDSON, H. E., C.I.E., C.B.E., 5 Kinburn Place, St. Andrews, Fife, Scotland.

§RICKLEFS, Dr. M. C., 16 Laurel Way, Ickleford, Hitchen, Herts.

RINDEN, Dr. A., 19 Robin Road, West Hartfort, Connecticut 06119.

ROBERTS, L. P., San Vio 866, 30123 Venice.

§ROBINSON. B. W., 41a Redcliffe Gardens, London S.W.10.

Robinson, G., Flat 10, Tranmere Court, Langley Park Road, Sutton, Surrey.

$\dagger$ ROBSON, Prof. J., 17 Woodlands Drive, Glasgow C.4.

ROCHER, Prof. L., The Dorchester, 1505, 226 W. Rittenhouse Square, Philadelphia, Pa. 19103.

Ronall, J. O., 333 Central Park West, New York N.Y. 10025.

ROQUE, Miss M. M., Carmelite Tertiary House, 20 Araneta Avenue, Quezon City, Philippines.

RosE, A.P., S.O.A.S., Malet Street, London WC1E 7HP.

RoSS, E. S., 35 Croftdown Road, London S.W.5.

ROTH, P., Barenburg, 5600 Lenzburg, Switzerland.

$\dagger$ ROTOURS, Baron R. des, 2 rue Joseph-Bertrand, 78 Viroflay, France.

Rouhani-PotTER, G. C., 142 Fairfield Street, Mount Hawthorne, Western Australia 6016.

ROWLANDS, H., 15 Rowan Road, London W.6.

ROY, Dr. K. K., 55 Gariahat Road, PO Box 10210, Calcutta 700019.

ROY, Prof. W. T., Dept. of Politics, University of Waikato, Hamilton, New Zealand.

§RUNCIM AN, the Hon. Sir Steven, Elshieshields, Lockerbie, Dumfriesshire.

RUSSELL, J. R., 3 Netherhall Gardens, London N.W.3.

RUSSELL, M., 62 Eaton Place, London S.W.1.

RUTNIN, Mrs. M., Thammasart University, Bangkok.

RYMER, D. T., Eastcombe House, Olveston, Bristol.

SAFADI, Y., Dept. of Oriental Printed Books \& Manuscripts, The British Library London W.C.1.

SAGGS, Prof. H. W. F., Brynteg House, Llantrisant, Gwent.

SAIDI, N., Flat 14, 94 Cornwall Gardens, S.W.7.

ST.MARTIN, M. O., c/o Midland Bank Ltd., 912 Brighton Road, Purley, Surrey. SALOMON, R., D.O.21, University of Washington, Seattle, Washington 98195.

SANDOR, Dr. E. E., 1 Fairlawn Drive, Woodford Green, Essex.

SANTA MARIA, J., 26 Balfen Grove, London W.4.

SANTUCCI, Dr. J. A., Dept. of Linguistics \& Religious Studies, California State 
University, Fullerton, Calif. 92634.

1970 Sarna, Prof. N. M., 35 Everett Street, Newton Center, Mass. 02159.

1973

1971

1969

1944

1972

1969

1977

1974

1955

1971

1954

1975

1972

1972

1972

1977

1974

1978

1975

1962

1974

1973

1933

1978

1957

1963

1976

1963

1968

1976

1973

1979

1946

1973

SATHAYE, S. G., 45 Victoria Road, Worthing, Sussex.

SATHYANARAYANA, Prof. R., 30 3rd Main, Jayanagar, Mysore 570014.

SAWERS, R. G., 5 South Villas, London N.W.1.

HoN. 1958 SCHAEFFER, Prof. C. F. A., Le Castel Blanc, 16 Rue Turgot, St. Germain-en-Laye, France.

SCHENK, N., Shady Lane, Monterey, Cal. 93940.

SCHNELLING, A., 14 Wildcroft Manor, London S.W.15.

SCHOFIE LD, Mrs. S. A., Casa Beaumont, VA TS 60, Javea, Alicante, Spain.

SCHRAPEL, Dr. D., Keisebackweg 12, 3553, Cölbe, West Germany.

†SCOTT, Sir M., KCMG., c/o Brooks's Club, St. James's, London S.W.1.

SEAGER, G. W., Bosphorus House, 28 Kippington Road, Sevenoaks, Kent.

SEGAL, Prof. J. B., S.O.A.S., Malet Street, London WC1E 7HP.

§SEIDEL, J. J., Jr., Mountain Road, Box 45, Hillsboro, Virginia, 22132, U.S.A.

SELKIRK, The Rt. Hon. The Earl of, K.T., P.C., G.C.M.G., G.B.E., A.F.C., Q.C., 60 Eaton Place London SW1X 8AT.

SELLWOOD, D. G. J., 44 Richmond Road, London SW20 0PQ.

SELTMAN, M. J. P., present address unknown.

SELWYN, J. B., 26 Fairway, Merrow, Guildford, Surrey.

SEN, Dr. N. D., 72 Hindusthan Park, Calcutta 29, India.

SENGUPTA, M. A., Prabhat Ranjam, 319/12, Jessore Road, Khudiram Colony, Calcutta 700074.

SENIOR, R. C., The Tower, Butleigh Court, Butleigh, Glastonbury BA6 8SA.

SERJEANT, Prof. R. B., Eastings, 99 High Street, Linton, Cambridge.

$\ddagger$ SERSEN, W. J., 587 West Merle Court, San Leandro, Calif. 94577.

SEVERIN, G. T., 74 Hemingford Road, London N.1.

†SEVIAN, V., 62A/1/34 Arasat Bahoshy, Alwiyah, Baghdad.

SEYFORT RUEGG, D., 15 Cadogan Square, London SW1X 0HT.

†SHAH, Prof. S. H., 8-5C (9-7-1-), Nr. Fire Brigade, Islamabad, Pakistan.

SHAKED, S., Institute of Asian \& African Studies, Hebrew University, Jerusalem.

SHARMA, Dr. B. N., 14, G.T. Road, Ghaziabad, U.P., India.

SHARMA, Prof. J. P., Dept. of History, 2550 Campus Road, University of Hawaii, Honolulu, Hawaii 96822.

SHASHTRI, K. C., 12B Dalim tala Lane, Calcutta 6.

SHAW, G. W., 85 Mordern Hill, London S.E.13.

ShBOUL, Dr. A. M. H., Dept. of Semitic Studies, University of Sydney, Sydney 2006.

SHEPHERD, Miss V., 13 Lymington Gardens, Ewell, Surrey.

SHEPPARD, Tan Sri Datuk Haji Mubin, P.S.M., DJPD., C.M.G., M.B.E., Kenangan 16 Road 9/3, Petaling Jaya, Selangor, Malaysia.

HON. 1978 SHERWANI, Prof. H. F., 602 Himayatnagar, Hyderabad 500029, India.

1954 §SHORTO, Prof. H. L., 5 Denny Crescent, London SE11 4UY.

1966

1954

1937

\$SHUJATUlLAH, S., 7 Colosseum Terrace, Albany Street, London N.W.1.

§SIMMONDS, Prof. E. H. S., S.O.A.S., Malet Street, London WC1E 7HP.

§SIMON, Prof. E. J.W., C.B.E., 13 Lisbon Avenue, Twickenham, Middlesex TW2 5HR.

1974 SIMS, Prof. E. G., 82 Cornwall Gardens, S.W.7.

1975 SIMS-WILliams, N. J., 38 Parolles Road, London N19 3RD.

1979 SINGER, J., 21 Perham Road, London W.14.

1964 SiNGH, Prof. K., Dept. of Punjabi Historical Studies, Punjabi University, Patiala, India.

$1933+$ †INGH, Sri K. N., Shri Sitaram Kunj, 1/B-1 Moti Lal Nehru Marg, (Lowther Road), Allahabad 2, U.P., India.

1972 SiNGH, Dr. P., Banares Hindu University, Varanasi-5, India. 
SINGH, Dr. R., D55197 Aurangabad, Varanasi-1, India.

SINGHAL, Dr. D. P., Dept. of History \& Political Science, University of Queensland, St. Lucia, Queensland 4067, Australia.

†SINH, Maharaj Kumar Dr. R., Raghubir-Niwas, Sitamau, Malwa, India.

†SINHA, G. C., B-909, Sector A, Mahanagar, Lucknow 226006.

$\dagger$ SinHA, K. K. P., Raio Kotla, P.O. Narki, Agra, India.

$\dagger$ SINHA, L. P. N., Bazaza Lane, Bakerganj, Patna 4, India.

$\S$ SINOR, Prof. D., Indiana University, Goodbody Hall, Bloomington, Indiana 47401.

SIRCAR, Prof. D. C., P. 645, Block O, New Alipore, Calcutta 53.

SIT APATI, Sri P., present address unknown.

SIUDMAK, J. E. C., c/o Christie's, 8 King Street, London S.W.1.

1975 SIVARAmamURTI, Dr. C., National Museum, Janpath, New Delhi-11.

SIV ARMAN, K., Tamilagam, 1500 South Rampart, Tanjore, Tamil Nadu, India.

§SKELTON, R. W., 22 Netherton Road, St. Margaret's, Twickenham, Middlesex.

$\dagger$ §SKILliter, Dr. S. A., 13 Brunswick Walk, Cambridge CB5 8DH.

SKINNER, Prof. C., Monash University, Victoria, Australia.

SKINNER, O. K., 438 Porlock Road, Honolulu, Hawaii 96825.

$\ddagger$ SKORUPSKI, T., 36 King Street, Tring, Herts.

SMART, Dr. E. S., c/o F. M. Golding Esq., 33 Upper Street, London, N.1.

SMITH, Prof. B. L., Carlton College, Northfield, Minnesota 55057.

$†$ SMITH, B. D., 78 Sherringham A venue, London N.14.

SMITH, D. J., Dept. of Religious Studies, Cartmel College, University of Lancaster, Bailrigg, Lancaster LA1 4YL.

SMITH, G. R., School of Oriental Studies, University of Durham, Elvet Hill, Durham DH1 3TH.

SMITH, J. D., 149 Gwydir Street, Cambridge.

SMITHIES, M. J., The University of Technology, PO Box 793, Lae, Papua New Guinea.

SNELL, R., S.O.A.S., Malet Street, London WC1E 7HP.

SNELlg ROVE, D. L., Restharrow, Cross Oak Road, Berkhamstead, Herts.

SNYDER, P. H., 321 Broadway, New York, N.Y. 10007.

SOKOLOFF, Mlle. V., 22 rue de Béarn, 92210 St. Cloud, France.

SOM, Dr. S. S., Parvati Krupa, 92 Ghantikeri Street, Hubli 580020, Karnataka, India.

SOMERS, G. E., Development \& Extension Dept., Lucknow Christian College, Lucknow 226001.

SOMERS, J. I., Flat 3 Buckley House, 95 Addison Road, London W.14.

SONI, Miss S., The Soni Building, "C" Road, Mandalay, Burma.

†SORCAR, "Padmashri" P.C., Indrajal, 276/1 Rash Behary Avenue, Ballyganj, Calcutta 19.

SOUL, J. B., "Speedwell", Kingsway, Bognor Regis, Sussex.

§SOUTHALl, E. P., 353 Hills Road, Cambridge CB2 2QT.

SPAAN, J. F. H., Minervaplein 36/3, Amsterdam.

†SPEAR, Dr. T. G. P., O.B.E., 29 Owlstone Road, Cambridge.

† SPEllmaN, Dr. J. W., Dept of Asian Studies, University of Windsor, Windsor, Ontario, Canada.

SPELMAN, D. P., 165 Pullman Court, London SW2 4SZ.

SPENCER, L. E., 27 Charles Hockling House, Bollo Bridge Road, London W.3.

SRINIVASAN, V., 27 Pila Tope, Mylapore, Madras 4.

†SRIVAstiva, Prof. R. P., Post Graduate Dept. of Fine Arts, Govt. College for Women, Pattala, Punjab, India.

†ST ANDISH, Comdr. J. F., The Oast House, Chequers Close, Crowborough, Sussex.

STARGARDT, Prof. A. W., 10 Southacre, Latham, Cambridge.

STARGARDT, Prof. J. M., 10 Southacre, Latham, Cambridge.

STEEDS, D., Dept. of International Politics, University College of Wales, Llandinam Building, Aberystwyth. 
1950 STERNBACH, Dr. L., 6 rue Leon Cogniet, 75017 Paris. $\nmid$ STONE, Mrs. L. H., Apt. 308, 55A Avenue Road, Toronto, Canada, M5R $2 Q 3$.

STRELCYN, S., Dept. of N. Eastern Studies, University of Manchester, Manchester 13.

1956 †TUUAR, Prof. D. G., present address unknown.

1968

1971

1966

1974

1925

STUMPF, Rev. K. L., O.B.E., 33 Granville Road, Kowloon, Hong Kong.

STURZ, Mrs. M. M., 32700 Coastsite Drive, 108 Palos Verdes Peninsula, Calif. 90274.

†STUtLEY, Mrs. M. E. L., The Beeches, Caerwys, Mold, Flintshire, N. Wales.

SUBRAMANIAN, Dr. A., c/o Sri P. V. Appusundaram, 1-93 West Street Palamarneri, Turukattupalli 613104, Tamil Nadu, India.

1962

1960

1977

1957

1970

1959

1964

1969

1972

† SUKET, The Raja of, Rangmahal Sundarnagar, Suket State, Punjab.

$\ddagger$ Sula

†SUlliV AN, Rev. H. P., c/o Vassar College, Poughkeepsie, New York 12601.

SUTHER LAND, Miss B. M., Nutford House, Brown Street, London W.1.

SUTTON, Group Capt. H. T., O.B.E., D.F.C., 86 Brancaster Lane, Purley, Surrey.

SWAN, R. K., Rathmore, Horsell Vale, Woking, Surrey.

SWARUP, Prof. B., 547A Maholi Ki Paur, Mathura 281001, UP, India.

$\dagger$ SWEENEY, S., Raithouse, Carrowbeg, Westport, Co. Mayo, Ireland.

SYKES, Mrs. S., 19 Henconner Avenue, Leeds L37 3NW.

SZNYCER, Prof. M., 2 rue Emile Faguet, 75014 Paris.

$\uparrow$ TAGgaR, Y., 2 Palace Green, London W.8.

$\ddagger$ TAHERI, Dr. A.-Q., 64 Townsend Lane, Harpenden, Herts.

TALBOT, H. D., present address unknown.

TAMBIMUTTU, P., 74 Foxbourne Road, SW17 8EW.

TAPPER, Dr. B., c/o W. H. Gordon, P.O. Box 1019, Ansonia Station, New York, N.Y. 10023.

TARLING, P. N., History Dept., University of Auckland, Private Bag, Auckland, New Zealand.

1975 TASBIHI, Dr. G. H., P.O. Box 195, Tabriz, Iran.

1977

1970

1971

1963

1973

1972

1972

1937

1967

1968

1961

1946

1974

1974

1958

1962

1965

1971

1938

TATE, Dr. R.F.S., Batiment C7, Appt. 61, Sonatiba Bellevue, Constantine, Algeria.

†THAKKUR, Dr. G. C., Sind A. Pharmacy, 375 Kalbadeir, Bombay 2.

THAPAR, B. K., Archaeological Survey of India, Janpath, New Delhi 11.

THOMAS, K. T., P.O. Box 145, Johore Bahru, Malaysia.

ThOMPSON, G. F. M. P., Kleinwort Benson Ltd., 20 Fenchurch Street, London E.C.3.

$\uparrow$ THOMPSON, M. S., 11 Old Ford View, Barnet, Herts.

$\ddagger$ THOMSON, Miss L. M., 3 Grove Terrace, London N.W.5.

$\dagger$ ThORNTON, J. S. D., 34 Victoria Road, Summertown, Oxford.

$\uparrow$ THURSBY, G. R., Box 13795, University Station, Gainesville, Florida 32601.

TIBBETTS, G. R., Chattesholt, Cassington Road, Eynsham, Oxford.

TITlEY, Miss N.M., Dept. of Oriental Printed Books and Manuscripts, British Library, London W.C.1.

TOMLIN, E. W. F., C.B.E., c/o Bank of Scotland, Haymarket, London S.W.1.

TOMLINSON, Sir Stanley, K.C.M.G., 6/24 Buckland Crescent, London N.W.3.

TOPSFIELD, A. S., 35 Ranelagh Gardens, Stamford Brook Avenue, London W.6.

TOVEY, Miss L., 6 Gossage Road, London S.E.18.

TRAUTMANN, T. R., Dept. of History, University of Michigan, Ann Arbor 48104 Michigan.

Treg ASKIS, M. M., 10 Waterside Plaza, Apt. 16-D, New York N.Y. 16010.

TREGEAR, Miss M., 29 Portland Road, Oxford.

$\dagger$ TR IMINGHAM, Rev. J. S., 72 Exmoor Drive, Worthing, Sussex.

1977

TRIPATHI, Mrs. U,, 24, Vectis Road, London SW17 9RG. 
1977 TRIVEDI, Dr. H. V., 33 Shankarbag, Indore 45002, Madhya Pradesh, India.

1972 TSUAN, Prof. T. H., 9 Glover Road, Milltown, New Jersey 08850.

HON. 1975 TUCCI, Prof. G., via Crocetta 8, S. Polo dei Cavalieri, Rome.

1965 TULAND, Dr. C. G., 8301 Mission Gorge Road, SP 166 Santee, Calif. 92071.

1944 TURK, F. A., "Shang-ri la", Reskadinnick, Camborne, Cornwall.

1962 TURNER, G. E., Overtoom 172, Amsterdam.

$1912 \dagger \S$ TURNER, Sir Ralph L., MC., Haverbrack, Barrells Down Road, Bishop's Stortford, Herts.

1966

$\dagger$ TWITCHETT, Prof. D. C., Faculty of Oriental Studies, Sidgwick Avenue, Cambridge.

1950 §ULlENDORFF, Prof. E., 4 Bladon Close, Oxford.

1969 UNGER, E. de,, The Manor House, Ham, Richmond, Surrey.

1967 VAIDYANATHAN, K. S., 4/617 Trichy Road, Coimbatore 19, Madras State, India.

VARMA, Dr. S. K., 9a/63 W.E.A., K. Bagh, New Delhi 5, 110005.

+VARMA, Prof. S., B-35 Defense Colony, New Delhi 24.

VAUDEVILLE, Prof. C., present address unknown.

VAUGHAN, D. A. J., 13 Phillimore Place, London W.8.

VAUGHAN, Mrs. P., Christie's, 8 King Street, London S.W.1.

VEITCH, Rev. J. A., 132 Sophia Road, Singapore 9.

VEJJAJIVA, V., Royal Thai Embassy, 30 Queens Gate, London S.W.7.

VERLOOP, Miss E. A., Paulinastraat 49, The Hague.

VERMA, Prof. V. K., Boulevard Lenin 125, Block 6, Apt. 2, Sofia.

†VINCENT, A.W., 44 Heathcliff Crescent, Balgowlah Heights, 2003 N.S.W. Australia.

1964 †VOGEL, Prof. Dr. C., 1 Londonerstrasse 11, 5300 Bonn.

1976

1953

1948

\$OYCE, M., 28 Balmore Street, London N.19.

De VREESE, Prof. Dr. K., Herengracht 256, Amsterdam.

†VYAS, Dr. K. B., Desai Chandulah Manilal, Arts \& Commerce College, Viramagam, Ahmedabad, India.

†WAARDENBURG, Dr. J. D. J., University of Utrecht, Institute of Theology, 2 Heidelberglaan, Utrecht, Holland.

1930 WadIA, Mrs. S., Theosophy Hall, 40 New Marine Lines, Bombay 1.

1972 †ADE, H. E., R. H., Embassy of New Zealand, 53 Bon-Bad, Turmstrasse 4, Godesberg, Germany.

1944 WAHBY, Colonel T., C.B.E., 7 Ferry Road, London S.W.13.

HON. 1958 W ALDSCHMIDT, Prof. Dr. E., Hainbundstrasse 21, 34 Göttingen, Germany.

$\uparrow$ \$ WLES, H. G. Quaritch, Oversted, Scotland Lane, Haslemere, Surrey.

WALEY-COHEN, Miss J., 11 Little St. James Street, London S.W.1.

WALKER, Lt.Col. J. G. B., 6 Grenville Mansions, Hunter Street, London W.C.1.

WARBURTON, Miss E. A. M., United Nations, Room 1229, New York N.Y., 10017.

WARE, Dr. L. L., 11 Old Square, Lincolns Inn, London W.C.2.

WARREN, J. C. T., Parsons Farm, Kerves Lane, Horsham, Sussex.

WASSON, R. G., 42 Long Ridge Road, Danbury, Conn. 06810.

WATSON, P. J., 15 Yew Tree Avenue, North Anston, Sheffield, S31 7EW.

§WATSON, Prof. W., Percival David Foundation, 53 Gordon Square, London W.C.1. 
1977 Whitaker, Capt. D. J., The Officers' Mess, 3rd Battalion Parachute Regt., B.F.P.O. 36, Germany.

1969 †Hitelaw, C. M. S., 29 Victoria Terrace, Crieff, Perthshire.

1973 WhITlEY, Mrs. E. M., Bromeswell Hollow, School Lane, Bromeswell, Woodbridge, Suffolk.

1976

1978

1978

1958

1974

1963

1978

1971

1977

1971

1976

1952

1968

WICKREMERATNE, Dr. L. A., 24 Rajapilhilla Mawatha, Kandy, Sri Lanka.

WHITE, C., S.J., Dept. of Philosophy \& Religion, The American University, Washington D.C., 20016.

WIGGINS, K. L., 33 Fermor Way, Crowborough, Sussex.

WILCOX, Rev. M., 84 Penrhos Road, Bangor, Wales.

WILDE, Miss G., 20 Castle Gardens, Swann Street, Petersfield, Hants.

WILEMAN, D., 62 Mary Green, Abbey Road, London N.W.8.

WILKES, C. M. P., 6 Durand Gardens, London S.W.9.

WILLEY, Major P. R. E., Wellington College, Crowthorne, Berks.

Williams, A. B., 5B Lyndhurst Gardens, London N.W.3.

WILliams, Miss A., Dept. of History, Kings College, Aberdeen AB9 2UB.

WILliAMS, D. J., 46 Lancaster Gate, London W.2.

WILKINSON, D. C., Glen Affric, 25 Manor Wood Road, Purley, Surrey.
WILSON, Dr. D. C., Political Adviser's Office, Central Government Offices, Hong Kong.

1967

WILSON, G, A., 10 Miterdale, Quaker Green, Woodthorpe, York.

WILSON, Col. J. E., 400 East 48th Street, New York N.Y. 10022.

WILsON, Prof. W. J., Oriental Studies, University of Arizona, Tucson, Arizona 85721.

WINDER, Miss M., 157 Queen Alexandra Mansions, Judd Street, London W.C.1. WINDER, Prof. R. B., 86 Castle Howard Court, Princeton, New Jersey 08540.

WINTER, Dr. H., 10 Ruth, 6095 Ginsheim, Germany.

WINTER, H. J. J., 39 Tollards Road, Countess Weir, Exeter, Devon.

WISSEMAN, Miss J., William Goodenough House, Mecklenburgh Square, London W.C.1.

WITTON, R. L., Blackdale, 178 Shirley Church Road, Shirley, Surrey.

WOLTERS, O. W., O.B.E., Dept. of History, Cornell University, Ithaca, New York 14853.

WolFF, Mrs. A. S., Fulwood Park, Liverpool 17.

WORTHINGTON, Dr. J. D., 2716 Coloma Street, Placerville, Calif. 95667.

WoODS, J., 83 Blaguegate Lane, Latham, Skelmersdale, Lancashire.

†WRIGHT, Prof. J. C., S.O.A.S., Malet Street, London WC1E 7HP.

$\ddagger$ WUJASTYK, D., Brasenose College, Oxford.

WYATT, Dr. N., Dept. of Religious Studies, The University of Glasgow, Glasgow G12 8QQ.

YADAV, Dr. K. C., Kurukshetra University, Kurukshetra, India.

YANG, W. L. Y., Seton Hall University, South Orange, New Jersey 07079.

YAR-SHATER, E., 450 Riverside Drive, Apt. 22, New York N.Y. 10027.

YORK, Mrs. G. H., 32 Overhill Drive, Patcham, Brighton, Sussex.

YOR KE, Dr. M., 12 Belsize Park, London N.W.3.

†YOUNG, M. J. L., Dept. of Semitic Languages \& Literature, The University, Leeds 2, Yorkshire.

1961 ZANDER, Dr. W., 16 Alvanley Gardens, London N.W.6.

1954 †OETMULDER, Prof. P. J. Gadjah Mada University, Jogjakarta, Indonesia.

1962 ZWALF, W., Shoe Cottage, Blunham, Beds.

1978 ZWANGIZER, Dr. R., Institut für Sprachwissenschaft, University of Vienna, Dr. Karl Lueger-Ring 1, A-1010 Vienna. 


\section{LIST OF LIBRARIES AND NON-MEMBERS SUBSCRIBING TO THE SOCIETY'S JOURNAL}

Aarhus, C., Denmark : Statsbiblioteket, Tidskriftafdelingen, Universitetsparken.

Aberdeen, Scotland: Periodicals Section, Aberdeen University, New Library, Meston Walk. Abo, 50, Finland: Steinerbiblioteket, 20500.

Adelaide, S. Australia, 5001: The Librarian, Barr Smith Library, University of Adelaide.

Agra, 282004, India: Mr. Y. Nath, c/o The National Book House, Jeonimandi.

Agra, 282004, U.P., India: K. M. Institute of Hindu Studies \& Linguistics, Agra University. Ahmedabad, 1, Gujerat, India: The Assistant Director of Archaeology, Dist. Panchayat Building, 5th Floor, Bhadra.

Ahmedabad, 9, India: Gujerat University Library, Nabrangpura.

Aichiken, 470-01, MZ, Japan: Aichi Gakuin Daigaku, Toshokan Iwasaki, Nisshin, Aichigun. Ajmer, 305001 Rajasthan, India: The Principal, Sowitri Girls College.

Akron, Ohio 44325, U.S.A.: Serials Department, Pierce Library, University of Akron.

Alabama 36112, U.S.A.: Air University Library, Serial Acquisitions, Building 1405, Maxwell Air Force Base.

Albany, N.Y., 12203 U.S.A.: Per. Sec. University Library, University of New York. 1400 Washington Avenue.

Albany, N.Y., 12230, U.S.A.: New York State Library, Serials Control Section A 388383002 , Cultural Education Center.

Albuquerque, New Mexico 87131, U.S.A.:

Aleppo, Syria: Periodicals Division, KEI, Aleppo University Library.

Alexandria, A.R.E.: University, Faculty of Arts, Chatby.

Alexandria, A.R.E.: University Central Library.

Aligarh, 202001 , U.P., India: The Librarian, Maulana Azad Library, Muslim University.

Allahabad, U.P., India: Hon. Librarian, General Library, University of Allahabad.

Allahabad, 21102 , U.P., India: Rajkeeya Public Library.

Ames, Iowa, 50010, U.S.A.: The Library, Iowa State University of Science and Technology. Amherst, Mass., 01003, U.S.A.: Serials Sections, The Library, University of Massachusetts. Amman, Jordan: The Library, University of Jordan.

Amritsar, 143001, India: The Head (4731) University Library, Guru Nanak University.

Amsterdam, 1001, Holland: Archaeologisch Instituut, Oude Turfmarkt 129.

Amsterdam, C., Holland: Universiteits-Bibliotheek, Singel 423.

Andhra Pradesh, India: Andhra University Library, Waltair, Visakhapatnam-530003, U.P.

Ankara, Turkey: Bibliothèque Nationale Milli Kutuphane, Department des Periodiques.

Ankara, Turkey: National Library, Namik Kemal Mahallesi, Kunsular Sokak No. 3.

Annamalaingar 608101, India: Annamalai University Library.

Anuradhapura, Sri Lanka: Public Library.

Aomori-ken 031, Japan (Kin): Hachenohe-Kosen Library, Att. Mr. Yoshiro Kodate, Uenodaira Tamogi.

Arlington, Texas 76019, U.S.A.: University of Texas at Arlington, Library Serials Department, P.O. Box 19218.

Ashkhabad, U.S.S.R.: Biblioteka Akademii Nauk, Ul. Gogolya 152.

Athens, Georgia 30602, U.S.A.: University of Georgia Libraries, Periodicals Desk-HY.

Athens, Ohio 45701, U.S.A.: Ohio University Library, Serials Department.

Atlanta, Georgia 30303, U.S.A.: Georgia State University, Library Periodicals Dept. 100 Decatier Street, S.E. 
Auckland, New Zealand: Serials Department, University of Auckland Library.

Austin, Texas 78712, U.S.A.: The Librarian, Serials Acquisition, University of Texas Library.

Baghdad, Iraq: Al-Mustansiriya University, AL/MUST/6/1682/77/30, The Library.

Baghdad, Iraq: Chief Librarian, University of Baghdad, Central Library, P.O. Box 12, Safa El Din at Hilli Street.

Baghdad, Iraq: The Library of Higher Studies, University of Baghdad Library, College of Arts.

Baghdad, Iraq: University of Baghdad, College of Law \& Political Science.

Bailrigg, Lancaster, England: Serials Department, University Library.

Balasore, Orissa, India: The Principal, Fakir Mohan College.

Baltimore, Md. 21204, U.S.A.: A. S. Cook Library, Towson State University.

Baltimore, Md. 21218, U.S.A.: Serials Department, The Library, Johns Hopkins University.

Bangalore 1, India: The Librarian, Bangalore University Library, P.O. Box 129.

Bangalore, 560-001, India: The British Library, 29, St. Mark's Road.

Bangkok, Thailand: Central Library, Thammasant University.

Bangkok, Thailand: National Library of Thailand, Samsen Road.

Bangor, N. Wales: The Librarian, University College of Wales, Gwynedd, LL57 2DG.

Barcelona, Spain: Biblioteca Catalunya, Calle Carmen 47.

Baroda, India: University Librarian, Shrimati Hansa Mehta Library, M.S. University of Baroda, (History), Station Road.

Basle, CH-4056 Switzerland: The Librarian, Oeffentliche Bibliothek der Universität, Zeitschriftenabteilung, Schöenbeinstrasse 18-20.

Baton Rouge, Louisiana 70803, U.S.A.: Lousiana State University, Library, Acquisitions Divisions, Serials Department.

Bawdigon, Burma: The Librarian (Oriental) and the Librarian (Eng.), Mandalay Arts \& Sciences University.

Beer Sheva, Israel: Ben Gurion University of the Negev, Library, P.O.B. 653.

Beirut, Lebanon: Arab University of Beirut, P.O. Box 5020.

Beirut, Lebanon: Bibliothèque Orientale, c/o P. Mech, 35 rue de Sèvres 75006 Paris, France.

Beirut, Lebanon: The Librarian, American University of Beirut, University Library Serials Dept., c/o Blackwell's Periodicals, Hythe Bridge Street, Oxford OX1 2EU.

Belfast, BT7 1LS, Northern Ireland: Queen's University Library.

Bellingham, Washington 98225, U.S.A.: Serials Dept., Wilson Library, Western Washington State College.

Benghazi, L.A.R.: Librarian, Central Library, Garyounis University, P.O. Box 1308.

Bergen, Norway: Universitetsbiblioteket i Bergen, Tidskrift kontoret 5000.

Berkeley, California 94709, U.S.A.: Pacific School of Religion, 1798 Scenic Avenue.

Berkeley, California 94720 , U.S.A.: Serials Department, University of California.

Berlin, Germany: Bruno Hessling, Buchhandlung, Rankestrasse 31-32.

Berlin, German Democratic Republic: Zeitungsvertriebsamt, Abt. Zentralvertrieb, Fruchstrasse 3-4, DDR-1004.

1 Berlin 30, West Germany: Staatsbibliothek, Preussischer Kulturbesitz, Erwerbungsabteilung, Postfach 1407 II Berlin, Reichpietsschufer 72/76.

1 Berlin 33 (Dahlem), Germany: Freie Universität Berlin, Orient. Institut, Abt. Semitistik und Arabistik, Reichensteiner Weg 8-10.

1 Berlin 33, Germany: Universitätsbibliothek, Freie Universität Berlin, Garystrasse 39.

Berrien Springs, Michigan 49104, U.S.A.: James White Library, Andrews University.

Bhubaneswar 4, Orissa, India: The Librarian, Utkal University Library, Vani Vihar.

Binghampton, New York 13901, U.S.A.: The Library, State University of New York at Binghampton, Vestal Parkway East.

Birmingham, B3 BHQ, England: The City Librarian, Reference Library.

Birmingham, B29 6LE, England: The Librarian, Selly Oak Colleges.

Blacksburg, Va. 24061, U.S.A.: Acquisitions Division, Virginia Polytechnic Institute \& State University, Carol M. Newman Library.

Bloomington, Ind iana 47401, U.S.A.: The Librarian, Indiana University. 
Bodh Gaya, Bihar State, India: The Professor in Charge, Department of Ancient Indian \& Asian Studies 4923, Mannuhal Magad University Library.

Bombay 400020, India: The Librarian, S.N.D.T. Women's University Library, Post Box No. 11163, New Marine Lines.

Bombay 400032, India: The University Librarian, University Library, Bombay University, Karmaveer Bhaurao Patil Marg, Fort.

Bonn, Germany: Der Bibliothekar, Universitätsbibliothek, Koblenzer Strasse 37/41.

Bonn, Germany: Universitätsbibliothek, Adenauerallee 39-41, D-5300.

Bonn 1, Germany: Messrs. H. Bouvier GmbH, Universitätsbuchhandlung, Postfach 1268, 5300 .

Boston, Mass. 02115, U.S.A.: Boston Museum of Fine Arts, 479, Huntingdon Avenue.

Boston, Mass. 02117, U.S.A.: Periodical Department, Boston Public Library, P.O. Box 286.

Boston Spa, Wetherby, Yorks. LS23 7BQ: The British Library, Lending Division.

Boulder, Colorado 80302, U.S.A.: University of Colorado Libraries, Serials Department.

D28 Bremen, 33, Germany: Universitätsbibliothek, Postfach 330160.

Bristol, BS8 1RJ, England: Bristo1 University Library, Wills Memorial Building, Queen's Road.

Bronxville 8, N.Y. 10708, U.S.A.: Sarah Lawrence College Library.

Brooklyn, New York 11238, U.S.A.: Brooklyn Public Library Periodicals Room, Grand Army Plaza.

Brooklyn, New York 11238, U.S.A.: The Librarian, Art Reference/Library, The Brooklyn Museum, Eastern Parkway.

Bryn Mawr, Pennsylvania 19010, U.S.A.: The Librarian, Bryn Mawr College Library.

Budapest 5, Hungary: Egyetemi Konyvtar, P.O.B. 483, 1372.

Buenos Aires, Argentina: Institute of Historia Astigua Oriental, Faculty of Philosophy \& Letters, 25 de Mayo 2173 piso, 1002.

Buenos Aires, Argentina: University of Salvador, School of Oriental Studies, Latin American Institute of Comparative Research, Sobre Callas 9660-1230.

Buffalo, New York, 14214, U.S.A.: Periodical Records and Bindery, Lockwood Memorial Library, State University of New York at Buffalo.

Buffalo, New York 14203, U.S.A.: Subscriptions Division, Buffalo and Erie County Public Library, Lafayette Square.

Bundoora, Victoria, Australia 3083: The Chief Librarian, Library, La Trobe University.

Burdwan, W. Bengal: The Librarian, Central Library, Galapbag, The University of Burdwan.

Caen, France: Bibliothèque Centrale, Service des Périodiques, 14032 Caen, cedex.

Cairo, Egypt, A.R.E., The Librarian, The American University at Cairo, The Library, 113 Sharia, Kasr el Aini.

Cairo, A.R.E.: Librarian, Ministry of Culture, The General Egyptian Book Organization, National Library and Archives.

Cairo, A.R.E.: Bibliothèque, Institut Francais d'Archaeologie Orientale, 37, Rue El Cheikh, Aly Youssef, (Rue Mounira).

Calcutta, India: Jadavpur University Library, Jadavpur.

Calcutta, India: State Central Library, 56A, B.T. Road.

Calcutta, 700073, India: The Librarian, Presidency College, College Street.

Calcutta 19, India: Dept. of Ancient Indian History and Culture, Calcutta University, 51/2, Hazra Road.

Calcutta 27, India: The Librarian, Government of India National Library, Belvedere, Alipore.

Calcutta 700050, India: Rabindra Bharati University, 56A Barrackpore Trunk Road.

Calcutta, 700029, India: Ramakrishna Mission, Institute of Culture, Col Park.

Calgary, Alberta, Canada, T2N 1N4: Periodicals Department, University of Calgary, 2920, 24th Avenue NW.

Calicut 1, Kerala State, India: The Professor \& Head of the Dept. of History, University of Calicut, Calicut University P.O. 673635.

Cambridge, England: The Librarian, Faculty Board of Oriental Studies, Sidgwick Avenue. Cambridge, CB2 3AP, England: The Master, Emmanuel College. 
Cambridge, Mass. 02138, U.S.A.: Harvard College Library, Serial Records.

Canberra, A.C.T. 2600, Australia: The Institute of Advanced Studies, Australian National University.

Canberra, A.C.T. 2600, Australia: Australian National Gallery.

Canterbury, Kent, CT2 7CN: The Library, The University.

Cape Coast, Ghana: The Librarian, University of Cape Coast, Private Post Bag.

Carbondale, Illinois 62901, U.S.A.: Southern Illinois University, Serials Department.

Cardiff, CP1 1XQ, Wales: The Main Library, Arts Periodicals, University College, P.O. Box 98, Corbett Road Bridge.

Chandigarh 14, India: The Librarian, Panjab University Library, Sector 14.

Chapel Hill, N. Carolina 27514, U.S.A.: Serials Department, University of N. Carolina, Chapel Hill, Wilson Library 024-A.

Charlotte, N.C. 28223, U.S.A.: Atkins Library, Serials Dept., University of N.C. Charlotte, UNCC station.

Charlottesville, Va. 22901, U.S.A.: Alderman Library, Serials/Periodicals, University of Virginia.

Charlottetown, Prince Edward I., Canada: Kelly Memorial Library, St. Dunstan's University. Charlottetown, Prince Edward I., Canada: The Librarian, University of Prince Edward Island. Chicago, Ill. 60611, U.S.A.: Public Library, Social Sciences \& History Division, 425 N. Michigan Avenue.

Chicago, Ill. 60680, U.S.A.: Serials Section, University of Illinois Library at Chicago Circle, Box 8198.

Chico, California, 95926, U.S.A.: Chico State College Library.

Chittagong, Bangladesh: Dept. of Oriental Languages, University of Chittagong.

Christchurch 1, New Zealand: The Librarian, University of Canterbury.

Cincinnati, Ohio 45202, U.S.A.: Cincinnati \& Hamilton County Public Library, 8th and Vine Streets.

Claremont, Calif. 91711, U.S.A.: The Honnold Library for Claremont College, Periodicals Dept.

Clayton, Victoria 3186, Australia: Periodicals Dept., Monash University Library.

Cleveland, Ohio 44114, U.S.A.: Cleveland Public Library, Order Dept., 325 Superior Avenue.

Cleveland, MS 38732, U.S.A.: Delta State University, W. B. Roberts Library, Periodicals Dept.

College Park, Maryland 20742, U.S.A.: The Librarian, McKeldin Library, University of Maryland.

College Station, Texas 77843, U.S.A.: Texas A. \& M. University Library, Serial Records.

Colombo, Sri Lanka: The Representative, The British Council, P.O. Box 753.

Colombo 7, Sri Lanka: Director of National Museums, Dept. of National Museums, P.O. Box 854.

Columbia, Missouri, U.S.A.: Library Serials Department, University of Missouri.

Columbia, S.C. 29208, U.S.A.: Serials Dept. Thomas' Cooper Library, University of S. Carolina.

Columbus 10, Ohio 43210, U.S.A.: Ohio State University Library, 1858 Neil Avenue.

Copenhagen, K., Denmark: The Librarian, The Royal Library, Christians Brygge 8, DK 1219.

Dacca, Bangladesh: Librarian, British Council, P.O. Box 161.

Damascus, Syria: Damascus University, Central Library, P.O. Box 3003.

Darien, Conn. 06820, U.S.A.: American Bibliographical Service, Box 1141.

Davidson, N.C. 28036, U.S.A.: The Librarian, Davidson College Library.

Davis, California 95616, U.S.A.: Acquisitions Department, The Library, University of California.

De Kalb, Ill. 60115, U.S.A.: Northern Illinois University, University Libraries, Periodicals Dept.

Delhi 110007, India: The Librarian, Delhi University Library, Periodicals Department.

Denver, Colorado 80210 , U.S.A.: Serials Division, University of Denver Library. 
Detroit, Michigan 48202, U.S.A.: Detroit Public Library, History \& Travel Department, 5201 Woodward Avenue.

Detroit, Michigan 48202, U.S.A.: Wayne State University, General Library, Serials Acquisitions Dept.

Dharwar 2, 580003, Karnatak State, India: Karnatak University Library, Chota Mahabaleshwar.

Dorking, Surrey RH4 3ET: Steel Bros. \& Co., Sondes Place.

Dunedin, New Zealand: The Librarian, Otago University Library.

Duntroon, A.C.T., 2600, Australia: Bridges Memorial Library, Royal Military College.

Durban, S. Africa: The Library, University of Natal, King George V Avenue.

Durham, England: University Library, Oriental Section, Elvet Hill.

Durham, North Carolina 27706, U.S.A.: The Librarian, Duke University Library, Periodicals D.S.

Düsseldorf 4000, Germany: Universitätsbibliothek, Zeitschriftenstelle, Universitätsstrasse 1.

East Lansing, Michigan 48823, U.S.A.: Michigan State University Library.

Edinburgh 8, Scotland: The Librarian, University Library, George Square.

Edmond, Oklahoma 73034, U.S.A.: Periodicals Dept., Central State University Library.

Edmonton T6G 2E1, Alberta, Canada: Acquisitions Division, Serials Section, University of Alberta.

Ehine-Ken 790 Japan: Matsujamashoka Daigaku, Toshokan, Bunkyo-cho, Maisuyama-Shi.

E1 Paso, Texas 79968, U.S.A.: University of Texas at El Paso, Library, Serials Dept.

Erlangen, Nürnberg, Germany: Universitätsbibliothek, Universitätsstrasse 4.

Essen/Lahn, Germany: Universitätsbibliothek, Bismarckstrasse 37.

Eugene, Oregon 97403, U.S.A.: The Librarian, University of Oregon.

Evanston, Illinois 60201, U.S.A.: North West University Library.

Flagstaff, Arizona 86011, U.S.A.: Acquisitions Librarian, C.U. Box 6022, Northern Arizona University Library.

Florence, Italy: National Library.

Florence, Italy: Universita degli Studi di Firenze, Biblioteca della Faculta di Lettere e Filosofia.

Folkestone, Kent CT19 5EE: Wm. Dawson \& Sons Ltd., Cannon House, Park Farm Road.

Freiburg-im-Breisgau D-7800, Germany: Karl Zink, Wissenschaftliche Buchimport, Sonnhalde 1191.

Freiburg-im-Breisgau D-7800, Germany: Universitätsbibliothek, Rempartstrasse 15.

Freiburg-im-Breisgau D-7600, Germany: Sprachwissenschaftliches Seminar, Universität Freiburg-im-Breisgau.

Fukuoka City, Japan: Kyushu University (Bungaku-Bu), Hakozaki.

Gainesville, Florida 32630, U.S.A.: University of Florida Libraries, Serials Department.

Geneva 4, Switzerland: Bibliothèque Publique et Universitaire, CH-1211.

Gettysburg, Pa. 17325, U.S.A.: The Librarian, Abdel Ros Wenz Library, Lutheran Theological Seminary.

Giessen/Lahn D-6300, Germany: Universitätsbibliothek, Bismarckstrasse 37.

Giza, A.R.E.: Cairo University Central Library, Periodicals Dept., Orman Gardens.

Giza, A.R.E. : Cairo University Library, Faculty Dar-el-Olum, Orman Gardens.

Glasgow G3 7DN, Scotland: The Librarian, Mitchell Library, North Street.

Glasgow G12 8QE, Scotland: The Librarian, Periodicals Dept., University Library.

Gondia, India: N.M.D. College Gondia, Dist. Bhandara, Maharashtra State.

Gorakhpur, U.P., India: The Honorary Librarian, University of Gorakhpur.

Göteborg 5, Sweden: Göteborgs Universitetsbibliotek, Allmänna Sektionen, Renstromgatan 4, Box 5096, S-402 22.

Göttingen D-3400, Germany: Niedersächsische Staats-und Universitätsbibliothek, Prinzenstrasse 1.

Greenville, U.S.A.: East Carolina College Library. 
Groningen, Netherlands: Bibliotheek der R.U., Semitistiek, P.O. Box 559.

Haifa, Israel: Per. Dept., Haifa University Library, Mount Carmel.

2-Hamburg 13, Germany: Staats-und Universitätsbibliothek, Moorweidenstrasse 40.

Hanover, New Hampshire 03755, U.S.A.: The Librarian, Baker Library, Dartmouth College.

Hayward, California 94542, U.S.A.: California State University, Hayward Library.

Heidelberg 1, D-6900, Germany: Kurt Ziehank, Postfach 1930.

Helsinki 17, Finland: University Library, PL312, SF 00171.

Hereford, England: Hereford College of Education.

Hiroshima, Japan: Hiroshima Daigaku Honbu, Toshokan/B/kai, Higashi-Senda.

Hobart, Tasmania: The State Librarian, State Library of Tasmania, 91 Murray Street.

Hobart 7001, Tasmania: The Librarian, University of Tasmania, Morris Miller Library, P.O. Box 252C.

Hong Kong: The Newspapers and Periodicals Section, City Hall Library, Edinburgh Place.

Hong Kong: The Library, University of Hong Kong, Pokfulam Road.

Hong Kong: The Librarian, Chinese University of Hong Kong, University Library, Shatin.

Honolulu, Hawaii 96822, U.S.A.: Serials Department, Asia Collection, University of Hawaii Library, 2550 The Mall.

Hounslow, Middlesex: Quaid-E-Islam University, Central Library, c/o Pakistan International Airlines, Cargo Development Manager, Cargo Terminal, Heathrow Airport.

Houston, Texas 77004, U.S.A.: Central Serials, University of Houston Libraries, 4800 Calhoun Road.

Hull, Yorks. HU6 7RX, England: The University, Brynmor Jones Library.

Hyderabad, Andhra Pradesh 500007, India: The Librarian, Osmania University College Library.

Hyogo-Ken, Japan: The Library, Kwansei Gakuin University, Uegahara, Nishinomiya-Shi.

Ibadan, Nigeria: The Librarian, Ibadan University Library.

Ibadan, Nigeria: The Librarian, Jos Campus Library, University of Ibadan, PMB 2084.

Ibaragi 30031, Japan: Tsukuba University Library, Viihari Gun.

Ile-Ife, Nigeria: The University of Ife Library.

Ilorin, Nigeria: The Librarian, University College Library, University College of Ilorin, PMB 1518.

Indiana, Penn. 15701, U.S.A.: Indiana University of Pennsylvania Library.

Iowa City, Iowa 52242, U.S.A.: The Librarian, University of Iowa Libraries, Serials Department.

Irvine, Calif. 92664, U.S.A.: The Serials Dept., The Library, University of California.

Isfahan, Iran: Central Library, University of Isfahan.

Ishikawa, MZ, Japan: Kanazawa University, Hobungkubu Library, Maranouchi 920.

Islamabad, Pakistan: The Librarian, Islamic Research Institute, Kendro Ramna 6, P.O. Box 1035.

Islamabad, Pakistan: The National Commission on Historical \& Cultural Research, Post Box 1230.

Islamabad, Pakistan: The Chief Librarian, University Islamabad.

Islamabad, Pakistan: The British Council, (for Islamabad Library, attention Chief Librarian), P.O. Box 1135.

Islamabad, Pakistan: The Representative, The British Council, P.O. Box 1135. (for Islamabad University, attention of Professor A. H. Dani, Director). Institute of Social Sciences.

Istanbul, Turkey: The Library, Bogazici Universitesi, Bebek, P.K.2.

Ithaca, New York 14853, U.S.A.: Central Serial Record Department, Cornell University Library.

Jaipur, Rajasthan, India: The Librarian, Rajasthan University Library, University Buildings. Jeddah, Saudi Arabia: Librarian, King Abdulaziz University, P.O. Box 3711.

Jerusalem, Israel: Periodical Department, Jewish National \& University Library, P.O. Box 503. 
Jerusalem, Israel: The Librarian, Ecole Biblique et Archéologique Française, P.O. Box $19 / 0053$.

Jodhpur 342001, Rajasthan, India: The Librarian, Jodhpur University Library.

Johannesburg, Transvaal, S. Africa: The Librarian, Johannesburg Public Library, Market Square.

Kabul, Afghanistan: The Representative, The British Council, P.O. Box 453.

Kabul, Afghanistan: Délegation Archéologique Française en Afghanistan.

Kagoshima 89101, Japan: Kagoshima Keizai Daigaku, Toshokan, Shimofukumoto-cho.

Kalamazoo, Michigan 49001, U.S.A.: Dwight B. Waldo Library, Serial Records, Western Michigan University.

Kampala, Uganda: Makerere University Library, Periodicals, P.O. Box 16002.

Kanagawa, Ken 259-12, Japan: Tokai Daigaku Toshokan, Chuo Lib. (NG) 117, Kitakanama Hiratsuka Shi.

Kano, Nigeria: The Librarian Abdullahi Bayero College Library, P.O. Box 453.

Kanpur 208001, India: Acharya Narendra Deva; Mahapalika Mahila Mahavidyalaya.

Karachi, Pakistan: The Librarian, University of Karachi, University Road.

Karachi, Pakistan: The Director, Dept. of Archaeology \& Museums, Govt. of Pakistan.

Karachi, Pakistan: The British Council, 50, Abdullah Haroon Road, P.O. Box 146.

Karlsruhe 1, West Germany: Badische Landesbibliothek, Postfach 1451, 75.

Karlsruhe 1, West Germany: Universitätsbibliothek Karlsruhe, Postfach 6920, 7500.

Kelaniya, Sri Lanka: The Librarian, University of Kelaniya, Vidyalankara Campus.

Kenosha, Wisconsin 53140, U.S.A.: Carthage College Library.

Kent, Ohio 44242, U.S.A.: Serials Dept., Kent State University Libraries.

Kiel, D-2300 Germany: The Director, Universitätsbibliothek. Zeitschriften u. TunschAbteilung.

Kingston, Ontario, Canada: The Librarian, Queen's University Library.

Kita-Kyoto, $603 \mathrm{MZ}$, Japan: Bukkyo Daigaku Toshokan, Kita Hananobo Murasakijo.

Knoxville, Tennessee 37916, U.S.A.: University of Tennessee Library, Serials Dept.

Köln, Germany: Universitätsbibliothek, Zeitschriftenstelle ORD 38, Universitätsstrasse 33.

Kuala Lumpur, Malaysia: National Archives \& Library, Tingkai, Wisma Sachdev, Jalan Raja Lant.

Kuala Lumpur, Malaysia: The Representative, The British Council, P.O. Box 539.

Kuala Lumpur, Malaysia: Serials Section, Acquisitions Services, University, Kebangs aan Malaysia, P.O. Box 1124.

Kuala Lumpur, Malaysia: Serials Division, University of Malaya Library, Pantai Valley.

Kuwait, Arab Gulf: Kuwait University, Libraries Dept., Periodicals Division.

Kyoto, $606 \mathrm{MZ}$, Japan: Bungakubu-Toshoshitsu, Kyoto Daigaku, Yosheda Sakyo-ku.

Kyoto $602 \mathrm{MZ}$, Japan: Library of Ohtani University, Karasuma-Gashira, Kamigyo-ku.

Kyoto $600 \mathrm{MZ}$, Japan: Ryukoku University Library, (Indo) Nanajyo-Inokuma, Shimogyo-ku.

Lagos, Nigeria: The Library, University of Lagos, Yaba.

Lagos, Nigeria: The Serial Section, The National Library, P.M.B. 12626.

Lahore, Pakistan: The Regional Representative, The British Council, 32 Mozang Road.

Lahore, Pakistan: Secretary, Dyal Singh Trust Library, Nisbet Road.

Laie: Oahu, Hi, U.S.A.: Learning Resource Center, Brigham Young University, Hawaii Campus.

Lausanne 1005, Switzerland: Bibliothèque Cantonale et Universitaire, Palais de Rumine.

Lausanne 1000, Switzerland: Librarie Centrale et Universitaire, S.A., 5 Place de la Palud, C.P. 404.

Lawrence, Kansas 66044, U.S.A.: Periodicals Section/Serials Dept., University of Kansas Libraries.

Leeds, Yorks., LS2 9JT, England: The Librarian, The Brotherton Library, University of Leeds, Woodhouse Lane.

Legon, Ghana: Balme Library, University of Ghana.

Leuven, Belgium: L. Wouters, Naamestraat 48, 3000. 
Lexington 29, Kentucky 115688 , U.S.A.: The Library, University of Kentucky.

Liège, Belgium: Section Orientale, Université de Liège, 16, Place du 20 Août.

Lincoln, Nebraska, 68508, U.S.A.: The Librarian, University of Nebraska, Acquisition Dept.

Liverpool L69 3DA, England: The University Library, P.O. Box 123.

London SW1Y 5ER, England: Secretary, Athenaeum Club, Pall Mall.

London, W.C.1., England: British Museum, Department of Western Asiatic Antiquities.

London WC1A 1AA, England: British Broadcasting Corp., Room 3, The Langham Hotel.

London WC1Y 2AA, England: The British Council, Periodicals Supply Section, Indonesia (Bandung), 65, Davies Street.

London, S.W.1, England: The Librarian, Foreign \& Commonwealth Office, Sanctuary Buildings, Great Smith Street.

London, W.C.1, England: Goldsmith's Librarian, London University.

London WC1E 7HU, England: Senate House, University of London, Malet Street.

London WC2H 8LP, England: Zeno, 6, Denmark Street.

London SE1 8NG, England: India Office Library, 197, Blackfriars Road.

London WC1H OPY, England: The Librarian, Institute of Archaeology, 31-34, Gordon Square.

London, S.W.1, England: The Librarian, London Library, 14, St. James's Square.

London, W.C.1, England: The Librarian, Warburg Institute, Woburn Square.

London WC2H 7HP, England: The City Librarian, Westminster Public Libraries, Central Reference Library, St. Martin's Street.

Long Beach, Calif. 90840, U.S.A.: California State University, Long Beach, Library Periodicals Dept., 1250 Bellflower Blvd.

Los Angeles, California 90041, U.S.A.: Occidental College Library, Periodicals Dept., 1600 Campus Road.

Los Angeles, California 90071, U.S.A.: Los Angeles Public Library, History, 630, West 5th Street.

Los Angeles, California 90032, U.S.A.: California State University, J. F. Kennedy Memorial Library, 5175 State University Drive.

Los Angeles, California 90007, U.S.A.: University of South California, Library, Serials Division, P.O. Box 77929.

Los Angeles, California 90024, U.S.A.: Serials Section, University Research Library, University of California.

Lubbock, Texas 79409, U.S.A.: Library, Periodicals Department, Texas Technological College, P.O. Box 4079.

Lucknow, U.P., India: The Honorary Librarian, Lucknow University, Tagore Library.

Lyon, Cedex 2, France: Bibliothèque Interuniversitaire de Lyon, 18, Quai Claude Bernard, 60365 .

Macomb, Ill. 61455, U.S.A.: 1085415, Serials Dept. Memorial Library, Western Illinois University.

Madison, Wisconsin 53706, U.S.A.: The Librarian, University of Wisconsin, Dept. of History, 3211 Humanities Building, 435 N. Park Street.

Madras 20, India: The Director, Adyar Library, c/o Research Centre, Theosophical Society, Adyar.

Madras 600 002, South India: Regional Education Adviser, British High Commission, British Council Division, 150-A, Anna Salai.

Madras 600008, India: The Librarian, Connemara Public Library, Egmore.

Madras 5, India: The Principal, Presidency College, Chepauk.

Madras, India: The Librarian, Madras University Library, Senate House, Triplicane.

Madrid 3, Spain: F. M. Pareja, Instituto Hispano-Arabe, Limite 5.

Mahwah, New Jersey 07430, U.S.A.: Rama Po College of New Jersey, Library.

Malegaon, Mahwashtra, India: Principal, MSG Arts, Science \& Commerce College.

Manasa Gangotri, Mysore 6, S. India: Mysore University Library.

Manchester M1 3 9PP, England: Periodicals Department, John Rylands University Library of Manchester, Oxford Road. 
Marburg/Lahn, Krummbogen 29, West Germany: Universitätsbibliothek Zeitschriftenselle D-355.

Markudi, Benne State, Nigeria: The Librarian, Murtala College of Arts, Science \& Technology. Marunouchi, Kanazawa, 920, Ishikawa, MZ, Japan: Hobungakubu Library, Kanazawa University.

Meerut, India: Digambar Jain College.

Melbourne, Victoria, Australia: The Librarian, State Library of Victoria, Swanston Street. Melbourne, Victoria 3000, Australia: National Museum of Victoria, 285-321 Russell Street.

Melbourne, Victoria, Australia: The Librarian, Baillieu Library, University of Melbourne, Parkville, N.2.

Memphis, T.N. 38112, U.S.A.: Burrow Library, North Western at Memphis.

Meshed, Iran: Central Library, University of Ferdowsi, P.O. Box 331.

Mieken 515, MZ, Japan: Maksuzaka Iyoshi Tanki Daigaku, Umemura, Kubo-cho, Matsusaka. Milano 20121, Italy: Biblioteca Nazionale Braidense, via Brera 28.

Milano 20123, Italy: Libreria Leđi, P. Cardorna 9.

Millersville, Pa. 17551, U.S.A.: State College, Periodicals, Ganser Library.

Milperra, N.S.W. 2214, Australia: Library, I.R.C. College of Advanced Education, P.O. Box 12.

Milwaukee, Wisconsin 53201, U.S.A.: Acquisitions Librarian, University of Wisconsin Library, Milwaukee, P.O. Box 604, 2311 , East Hartford Avenue.

Milwaukee, Wisconsin 53233, U.S.A.: Marquette University, 1415, West Wisconsin Avenue. Milwaukee, Wisconsin 53233, U.S.A.: Milwaukee Public Library, 814, W. Wisconsin Avenue. Minden, Penang, Malaysia: University Library, Universiti Sains Malaysia.

Minneapolis, Minn. 55455, U.S.A.: Serial Records, University of Minnesota Libraries.

Mississippi 39762, U.S.A.: Mitchell Memorial Library, Mississippi State University, State College.

Miyazaki, 880 Japan: Miyazaki Daigaku, Kyoiku-Gakuba Lib, Funazuka-cho.

Miyazaki, 800, Japan: Miyazaki University, Kyoikugakubu Library, Funazuka 1-chome.

Modasa 383315, Gujerat, India: The Principal, Shri S. K. Shah \& o.m. Arts College.

Monkton, Maryland 21111, U.S.A.: Mr. Alexander B. Griswold, Breezewood, 3722 Hess Road.

Montreal, 112, Quebec H3A 1Y1, Canada: Periodicals Department, McGill University Library, 3459, McTavish Street.

Montreal, Canada: Library-Serials, Concordia University, Sir George Williams Campus, 1455 , De Maisonneuve Boulevard West.

Montreal 101, Canada: Université de Montreal, Bibliothèque des Sciences Sociales, Salle des Périodiques, C.P. 6128.

Moscow V418, U.S.S.R.: Biblioteka Akademii Nauk Krasikova 2845, SSSR.

Mount Pleasant, Michigan 48859, U.S.A.: Central Michigan University, Library, Serials Dept. Munich, 40, Germany: Buchhandlung J. Kitzinger, Schellingstrasse 25, D-8000.

Munich, 34, Germany: Bayerische Staatsbibliothek, Zeitschriftensaal, Postfach 150, D-8000. Münster, Westf. 2, Germany: Antiquariat Th. Stenderhoff \& Co., Alter Fischmarkt 21. D44. Murdoch, 6153 Australia: Serials Section, Murdoch University Library.

Murray, Kentucky 42071, U.S.A.: Library Serials Dept., Murray State University.

Mussourie, Dist. Dehra Dun, India: The Librarian, Lal Bahadur Shastri National Academy of Administration.

Muzaffarpur, India: University of Bihar Library, P.O. Box 48.

Nagasaki 852, Japan: Nagasaki Daigaku Kyoikugakuba, Bunkyo Machi.

Nagoya, 464, Japan: Nagoya University Library, Furo-cho, Chikusa-Ku (KIN).

Nagpur, 440001, India: The Librarian, Nagpur University Library, North Ambazari Road.

Nagpur, 440001, India: Superintendent Epigraphist, Archaeological Survey of India, Excavation Branch, Old High Court Building.

Nankang, Taipei, Republic of China: The Library, Akademia Sinica, Institute of History and Philology.

Nedlands, W. Australia 6009: Reid Library, University of Western Australia. 
Nendeln, Liechtenstein: Kraus Periodicals, Subscription Dept. 47349 FL 9491.

Neuchâtel, Switzerland: Bibliothèque centrale de l'Université, CH-2000.

Newark, Delaware, 19711, U.S.A.: University Library, University of Delaware.

New Brunswick, New Jersey 08901, U.S.A.: Periodicals Dept., Rutgers University Library.

Newcastle, N.S.W., 2308 Australia: The Auchmuty Library, University of Newcastle.

New Delhi 1, India: The British Council Library, AIFACS Building, Rafi Marg.

New Delhi 11, India: The Assistant Librarian, Nehru Memorial Museum and Library, Teen Murti House.

New Delhi 110021, India: The Principal, Atma Ram, Sanatan Dharam College, Dhanla Kann.

New Delhi 110057, India: Officer on Special Duty (Library), Jawaharlal Nehru University, 22, New Mehrauli Road.

New Delhi 110025, India: Librarian, Dr. Zakir Husain Library, Jamia Millia Islamia, Jamia Nagar.

New Delhi 27, India: Shyama Prasas Mukherji College (for Women), Tagore Garden.

New Delhi 110016, India: National Council of Educational Research \& Training, N.I.E. Library, Documentation \& Information Services, Sri Aurobindo Marg.

New Haven, Conn. 06520, U.S.A.: Yale University Library, Serials Division.

New York, N.Y. 10027, U.S.A.: Columbia University Libraries, Serials Acquisition, 535, West 114th Street.

New York, N.Y. 10027, U.S.A.: Encyclopaedia Persica, 710 Hamilton Hall, Columbia University.

New York 10031, N.Y., U.S.A.: City College Library, (Periodicals Division), Convent Avenue and 135th Street.

New York, N.Y. 20028, U.S.A.: The Librarian, Metropolitan Museum of Art.

New York, N.Y. 12561, U.S.A.: Sojourner Truth Library, State University College, New Paltz.

New York, N.Y. 10017, U.S.A.: New York Public Library, Division O, Grand Central Station, P.O. Box 2231.

New York, N.Y. 11432, U.S.A.: The Queensborough Public Library, Acquisitions Division, 89-11 Merrick Boulevard, Jamaica.

New York, N.Y. 10021, U.S.A.: The Librarian, Union Theological Seminary, Haverford College, 3041 Broadway at 120 th Street.

New York, N.Y. 10012, U.S.A.: New York University, Elmer Holmes Bobst Library, T.S.D. Serials Dept., 70, Washington Square S.

Nijmegen, Holland: Universiteitsbibliotheek, Bestelafdeling, 1399A, 119. 884 Erasmuslaan 36.

Nijmegen, Holland: Universiteitsbibliotheek, 1600, A10, 908, Erasmuslaan 36.

Normal, Illinois 61761, U.S.A.: Serials Department, Illinois State University Library.

Norman, Oklahoma 73069, U.S.A.: Room 134, Serials Department, Library, 401, Brooks Street, University of Oklahoma.

North Ryde, N.S.W., 2113, Australia: Macquarie University Library.

Northampton, Mass. 01060, U.S.A.: Smith College Library.

Northfield, Minnesota 55057, U.S.A.: Library, Carleton College,-310.

Northridge, California 91330, U.S.A.: The Library, California State University, 18111 Nordhoff Street.

Notre Dame, Indiana 46556, U.S.A.: Serials Record (26397a), Memorial Library, University of Notre Dame.

Novosibirsk 200, U.S.S.R.: Gosudarstvennaya Publichnaya, Nauchneteknickeskaya Bibliotek, Pri. Sib. Otd. SSR Knigoobman.

Oakdale, New York, 11769, U.S.A.: Dowling College Library.

Oberlin, $\mathrm{OH} \mathrm{44074,} \mathrm{U.S.A.:} \mathrm{The} \mathrm{Librarian,} \mathrm{Oberlin} \mathrm{College} \mathrm{Library.}$

Okayama, 700, MZ, Japan: The Library, Okayama University, Tsushima.

Olney, Bucks. MK46 4BN: J.S.A. Garyounis, 4 Midland Road.

Olney, Bucks. MK46 4BN: J.S.A. Oxcutter, Patna University Library, 4 Midland Road.

Olney, Bucks. MK46 4BN: J.S.A. Riyad, 4 Midland Road. 
Omaha, Nebraska 68182, U.S.A.: Periodical Dept., Library, University of Nebraska, 60th and Dodge Streets.

Osaka, 565 Japan: 3415011 National Museum of Ethnology, Senri Expo Park, Yamada, Suira-Shi.

Osaka, 560 MZ, Japan: Osaka University, Bungokuba, Shiryoshitsu, Machikaneyama, Toyonaka.

Osaka, 567, Japan (Kin): Ottemon Daigaku Library, $230 \mathrm{Aj}$ Ibaraki-Shi.

Osakafu, 583, MZ, Japan: Shitennoji Women's College Library, 1308, Haniuno, Habikino-Shi.

Oslo 2, Norway: The Librarian, Universitets biblioteket, Tidskrifskont, Drammensvn 42B.

Oslo 3, Norway: UB's Bibl. T. JHF, Into-Iransk Inst., Boks loog-Blindern.

Ottawa, K1N 9A5, Canada: Morissett Library, Serials Currer, University of Ottawa.

Ottawa, Ontario KI8 5B6, Canada: Carleton University, Colonel By Drive.

Oxford, OX1 2PH, England: The Librarian, Ashmolean Museum.

Oxford, England: The Librarian, Indian Institute, Broad Street.

Oxford, OX1 2EU: Oriental Institute Library, Pusey Lane.

Oxford, OX1 2EU: University of KIAB/MECCA, c/o P.O. Box 40, Hythe Bridge Street.

Oxford, OX1 2EU, England: Blackwell's Periodicals Division, UAEU P.O. Box 40, Hythe Bridge Street.

Panaji 403401, Goa, India: The Director, Centre of Post-Graduate Instruction and Research, University of Bombay, Susheela, 18th June Road.

Paris VIIe, France: Bibliothèque de l'Institut Nationale des Langues et Civilizations Orientales 2, Rue de Lille.

Paris 75006, France: Bibliothèque Orientale/Beirut, c/o P. Mech, 35 rue de Sevres.

Paris, 75006, France: Librarie d'Amerique et d'Orient, Adrien-Maisonneuve, 11, Rue SaintSulpice.

Paris, 75270, France: Prof. Madeleine Biardeau, Centre d'Etudes de l'Inde et de l'Asie du Sud, 54, Boulevard Raspail.

Paris, 75260, France: Centre de Documentation, Sciences Humaines du C.N.R.S., B.P. 140. 06.

Paris VIe, France: Bibliothèque de l'Institut de France, 23, Quai de Conti.

Paris Ve, France: Bibliothèque de l'Université de la Sorbonne, 47, Rue des Ecoles.

Paris, 75084, France: Department Periodiques, Bibliothèque Nationale, 58, Rue de Richelieu.

Patna, Bihar, India: Sinha Library, Bihar State Central Library, P.O. Box No. 62, G.P.O.

Peking, China: O. No. 305C 04: DNS: 74, China National Publications, Import Corp., Periodicals Dept., P.O.B. 50.

Peking 7, China: International Exchange Section, The National Library of Peking.

Pennsylvania 16802, U.S.A.: Periodicals Section, Pattee Library, Pennsylvania State University, University Park.

Pensacola, Florida 32504, U.S.A.: Library Serials, University of West Florida.

Peradeniya, Sri Lanka: The Librarian, University of Sri Lanka, Perediniya Campus.

Philadelphia, Pa. 19151, U.S.A.: Eastern Baptist Theological Seminary, The Library, Periodical Division, City Line and Lancaster Pike.

Philadelphia, Pa. 19103, U.S.A.: Serials Section, Free Library of Philadelphia, Logan Square.

Philadelphia, Pa 19122, U.S.A.: Temple University Library, Serials Records Unit-Per.

Philadelphia, Pa. 19104, U.S.A.: The Library, University of Pennsylvania.

Pittsburgh, Pa. 15260, U.S.A.: Acquisitions Dept., Hillman Library, Pittsburgh University.

Pittsburgh, Pa. 15206, U.S.A.: Pittsburgh Theological Seminary, 616, Clifford E. Barber Library, Highland Avenue.

Plattsburg, New York 12902, U.S.A.: Benjamin F. Feinberg, Library, State University College, Beekman Street.

Pocatello, Idaho 83201, U.S.A.: Idaho State University Library, Serials Department.

Pondicherry 605601, India: The Pondicherry Museum, 1 rue Romain Rolland.

Poona 4, India: Professor-in-Charge, Bai Jerbai Wadia Library, Fergusson College.

Poona 7, India: Centre of Advanced Study in Sanskrit, University of Poona, Near Arts Faculty, Ghaneshkhind. 
Portland, Oregon 97207, U.S.A.: Portland State College, Library Serials, P.O. Box 1151.

Pretoria, S. Africa: The Librarian, Merensky Bibliotek, University of Pretoria.

Princeton, New Jersey 08640, U.S.A.: Speer Library, Princeton Theological Seminary, Box 111.

Princeton, New Jersey 08640, U.S.A.: The Librarian, Serials Division, University Library.

Princeton, New Jersey 08640, U.S.A.: Prof. B. Lewis's Research A/C, New Euston Studies, Princeton University.

Providence 02912, Rhode Island, U.S.A.: The Librarian Serials Department, Brown University.

Puerto Rico, 00931, U.S.A.: Sala de Revistas, Biblioteca Jose M. Lazaro, Universidad de Puerto Rico, Rio Pedras.

Queensland 4810, Australia: The Library, James Cook University of North Queensland, P.O. Box 999, Townsville.

Querenberg, West Germany: Universitätsbibliothek Bahum, Universitätstrasse 150, 4630 B.O.-Bochum.

Rabwah, West Pakistan: Khilafat Library, Sadr Anjuman, Ahmadiyyeh.

Raja Rammohunpur, W. Bengal, India: The Deputy Librarian, University of North Bengal.

Rajasthan, India: Central Library, Banasthali Vidyapith.

Rajkot, Gujerat, India: Saurashtra University Library, University Campus, Kalawad Road.

Raleigh, N. Carolina 27650, U.S.A.: Periodical Service Centre, D. H. Hill Library, P.O. Box 5007, N. Carolina State University.

Ramachandrapuram, (E.G. District), A.P., India: Principal (108), V.S.M. College.

Ramat Gan, Israel: Bar Ilam University Central Library.

Rannagar, Varanasi, India: American Institute of Indian Studies, Chief Court House.

Rangoon, Burma: The Librarian, University Central Library, University Estate.

Rawalpindi, Pakistan: Christian Study Centre, 126B Muree Road.

Rawalpindi, Pakistan: The British Council Representative, P.O. Box 1135, Islamabad.

Reading, Berkshire RG1 1TG. Alan Armstrong \& Associates Ltd., 13041-ARAMCO, 8 Queen Victoria Street.

Regensburg 2, Germany: Universitätsbibliothek, Postfach 409, Universitätstrasse 33.

Richardson, Texas, U.S.A.: University of Texas at Dallas, Library, P.O. Box 643.

Richmond, Indiana 47374, U.S.A.: Lilly Library, Earlham College.

Richmond, Virginia 23227, U.S.A.: Union Theological Seminary, 3401, Brook Road.

Riverside, Calif. 92507, U.S.A.: University of California Library, P.O. Box 5900.

Riyadh, Saudi Arabia: University of Riyadh (Per) c/o Box 40 Hythe Bridge Street, Oxford OX1 2EU.

Rochester, NY 14627, U.S.A.: Periodicals Dept., University of Rochester Library, River Campus Station.

Rome, Italy: Biblioteca Nazionale Centrale, Vittorio Emanuale II, via le Castro Pretorio.

Rome, Italy: Biblioteca Istituto Italiano per il Medio e Estremo Oriente, via Merulana 248.

Rye, NY 10580, U.S.A.: Mr. Sheldon Swartz, 58, Barlow Lane.

Saarbrïcken 11, Germany: Universitätsbibliothek, Zeitschriftenstelle, Universität des Saarlandes.

Sabah, Malaysia: Regional Representative, The British Council, P.O. Box 746, Kota Kinabalu.

Sahibabad, U.P., India: The Librarian, Inst. of Indology, Lajpai Rai College.

St. Andrews, Scotland: The Librarian, University Library.

St. Catherine's, Ontario, Canada L25 3A1: Brock University. Library, Decew Campus

St. Johns, Newfoundland: M.U.N. Library, Memorial University of Newfoundland.

St. Louis, Missouri 63130, U.S.A.: Washington University Libraries, Serials Department.

St. Louis, Missouri, 63108 U.S.A.: Pius XII Memorial Library, Saint Louis University, 3655 West Pine Boulevard.

St. Louis, Missouri 63105, U.S.A.: Library, Concordia Seminary, 801 De Mun Avenue. 
St. Lucia Queensland 4067, Australia: The Main Library, The University of Queensland.

St. Paul, Minn. 55108, U.S.A.: Luther-Northwestern Seminary Library, 2375 Como Ave.

Saitama-ken, 350-02, Japan: Josai Daigaku, Toshokan (Keizai Kyoyo), Tawama Sakadomachi.

Salisbury East, Australia 5109: The Library, College of Advanced Education, Smith Road.

Salt Lake City, Utah 84112, U.S.A.: Serials Order Dept., University of Utah Library.

Sambalpur, Orissa, 768017, India: Sambalpur University, Central Library, Jyoti Vihar, P.O. Burla.

San Diego, Calif. 92182, U.S.A.: Orders and Periodicals Serials, Malcolm A. Love Library, San Diego State University.

San Diego, La Jolla Calif. 92023, U.S.A.: Serials Records, Acquisitions Department, Library California State University at San Diego.

San Francisco, Calif. 94116, U.S.A.: Chinese Materials Center, Inc., 909, Taraval Street.

San Francisco, California 94102, U.S.A.: Periodical Department, San Francisco Public Library, Civic Center.

San Francisco, Calif. 94143, U.S.A.: The Library, Acquisitions Dept. University of California.

San Jose, Calif. 95192, U.S.A.: Library Serials Department, San Jose State University, 250, South 4th Street.

Santa Barbara, Calif. 93106, U.S.A.: Serials Library, University of California.

Santiniketan, W. Bengal, India: Visva Bharati Central Library.

Sapporo, 060, Japan: Hokkaido Daigaku, Bungakubu Toshoshitsu, Nishi-7 Kita 10.

Saskatoon, Saskatchewan S7N OWO, Canada: Murray Memorial Library, Serials Department, University of Saskatchewan.

Seattle, Washington 98105, U.S.A.: Acquisitions Division, University of Washington Library. Sendai, 980, Japan (KIN): Bungaku-bu Library, Tohaku University, Kawauchi.

Seoul, Korea: The Representative, The British Council, c/o British Embassy, BFPO 3.

Seoul, Korea: Chung Ang University, Central Library, Huksak-Dong, Kwanak-ku.

Seoul, Korea: Mr. Choi Chan-Kyun, Korea Overseas Publications Inc., P.O. Box 2558.

Seoul, Korea: Mr. Lee Man-Kap, Kwang Hwa Moon, P.O. Box 595.

Seoul, Korea: Mr. Lee Sun-kung, Kwang Hwa Moon, P.O. Box 1480.

Seoul, Korea: Yonsei University Library, 134 Shin Chin Dong.

Shanghai, China: Miss Wang Wen Juan, P.O. Box 4015.

Shillong, 793003, India: The Librarian, North Eastern Hill University, Mayurbhanj House, Nongthy mai.

Shiraz, Iran: Shiraz University, College of Arts and Sciences, Science Library.

Shiraz, Iran: The Regional Director, The British Council, P.O. Box 65.

Simla, India: Indian Institute of Advanced Study.

Sind, Pakistan: Sind University Central Library, Jamshore.

Singapore 3, The Librarian, Institute of Education, Paterson Road.

Singapore 6: The Director, National Library, Serials Sections, Stamford Road.

Singapore 10: The Librarian, University of Singapore, Bukit Timah Road.

South Orange, N.J. 07079, U.S.A.: Periodicals Dept., Seton Hall University, McLaughlin Library, attn.

Stanford, California 94305, U.S.A.: Serial Records, Stanford University Library.

Stillwater, Oklahoma 74074, U.S.A.: The Library, Serials Department, Oklahoma State University.

Stockholm 5, Sweden: Kungl. Biblioteket, Box 5039, S 102, 41.

Stony-Brook, L. I., N.Y. 11794, U.S.A.: The Institute for Advanced Studies of World Religions, 5001, Melville Memorial Library, State University of New York.

Storrs, Connecticut 06268, U.S.A.: W. Cross Library, University of Connecticut.

Strasbourg, France: Bibliothèque Nationale et Universitaire, 6, Place de la République.

Stuttgart, West Germany: Würtembergische Landesbibliothek.

Swarthmore, Penn. 19081, U.S.A.: College Library.

Sydney, NSW 2000, Australia: The Librarian, Public Library of New South Wales, Macquarie Street.

Sydney NSW 2006, Australia: The Librarian, State Library of New South Wales, Macquerie Street. 
Syracuse, 10, New York, U.S.A.: Syracuse University Library, Serials Division.

Szeged, 6701, Hungary: Jate Kozponto Kenyvtara.

Taipei, Republic of China: Library, National Taiwan Normal University, Ho Ping E Road See 1.

Taipei, Republic of China: Prof. Zu-Zan Yang, Main Library No. GL 7798, National Taiwan University.

Taipei, Republic of China:: Periodicals Dept., National Central Library, 433 Nan Rai Road, 107.

Taipei, Republic of China: CH Weng Wen Publishing Co., P.O. Box 22605.

Talence-Pessac, 33400 France: Bibliothèque de l'Université de Bordeaux, Section des Lettres et Sciences Humaines, Domaine Universitaire, Ave des Arts.

Tallahassee, Florida 32306, U.S.A.: Serials Division, Robert Manning Strozier Library, Florida State University.

Tampa, Florida 33620, U.S.A.: Acquisition Librarian, University of South Florida.

Tehran, Iran: Librarian, Asian Cultural Documentation Centre for UNESCO, 1-34 Iraq Street, Koorosh-e-Kabir Ave.

Tehran, Iran: The Representative, The British Council, P.O. Box No. 1589.

Tehran, Iran: Dr. F. Daftary, Saltanatabad Ave., 4th Golestan Street, No. 5.

Tehran, Iran: Central Library \& Documentation Centre of Tehran University.

Tehran, Iran: Free University of Iran, 101, Kakh Shomali Street, Shah Reza Avenue, P.O. Box 14-1972.

Tehran, Iran: Iranian Academy of Philosophy, Aryamehr University, Eisenhower Avenue.

Tel-Aviv, Israel: Tel Aviv University Library, P.O. Box 39038.

Tenri-Shi, Nara-ken 632, Japan: Tenri Toshokan (Yushodo), 1050, Semano-uchi-cho.

Thunder Bay, P 517502, Ontario, Canada: Lakehead University, Library, Serials Dept.

Tirupati, Andhra Pradesh, India: The Assistant Librarian, Sri Venkateswara University Library, P.O. Box 117.

Tokyo, Japan: Aoyama-Gakuin-Daigaku-Toshokan, Shibuya-4-Chome, Shibuya-ku.

Tokyo, Japan: Asia-Keiza-Kenkyujo, Ichigaya-Hon, Mura-cho, Shinjuku-ku.

Tokyo, Japan: Asia University Library, Sakia, Mushashino-Shi.

Tokyo, Japan: Bukkyogaku-Kenkyushitsu, Rissho University, Higashi-Ohsaki-4-Chome, Shinagawa-ku.

Tokyo 181, Japan: International Christian University Library, Osawa Mitaka-Shi.

Tokyo, Japan: Kokkai-Toshokan, Tosho-Kyoku, Nagatacho Chivoda.

Tokyo, Japan: Komazawa University Library, 1-23 Komazawa, Setagaya-ku.

Tokyo, 602, Japan: Library of Ohtani University, Karasuma-Gashira, Kamigyo-ku.

Tokyo, 101, Japan: Meiji University, Daigakuin-Tosheshitsu, 1-1, Kanda-Surugadai, Chiyoda-ku.

Tokyo, Japan: Nippon University, Bunrigaku-Bu Library (History), 25-40, Sakuru-Josu, 3-chome.

Tokyo, Japan: Ochanomizu-Joshi-Daigaki-Toshokan (Ochanomizu Women's University Library) Bunkyo-ku.

Tokyo, Japan: Rikkyo University, Bunkakubu-bu, Tosho Centre, Yoyoshi, Ikebukuro, Toshima-ku.

Tokyo, Japan: Sanseido Bookstore Ltd., Attn. A. Aoken, 1-1, Jimbocho, Kanda, Chiyoda-ku.

Tokyo, Japan: Taisho University, Toshokan Ukeire-gakari, No. 53 Nishisugamo 4, Toshima$\mathrm{ku}$.

Tokyo, Japan: Tokyo-Gaikokugo Daigaku-Toshokan (Tokyo University of Foreign Languages), Nishiga-hara-machi-, Kita-ku.

Tokyo, Japan: Toyo-Daigaku-Toshokan Zashishitsu (Tokyo University Library), Hakusan-5chome, Bunkyo-ku.

Tokyo, Japan: Acquisitions Sections, University of Tokyo Library, 3-1 Hongo 7-chome, Bunkyo-ku, 113.

Tokyo, Japan: Waseda University Library, Kohusaibu (Nakahara), 1-6-1, Nishi Waseda, Shinjuku-ku. 
Tokyo, Japan: Todai-Sogo-Toshokan, Hongo, Bungkyo-ku 113.

Toronto M5S 1A5, Canada: The Librarian, Serials Department, University of Toronto.

Toyohashi 440, Japan MZ: Aichi University Library, Machihata-cho.

Trivandrum, 695001, India: The Librarian, Kerala University Library.

Tübingen, Germany: Universitätsbibliothek, P.O. Box 149.

Tucson, Arizona 85721, U.S.A.: The Librarian, University of Arizona.

Tunis, Tunisia: Bibliothèque Nationale, Sec. Périodiques, BP 42.

Turin, Italy: Rosenberg \& Sellier, via Andrea Doria 14, 10123.

Ujjain, Madhya Pradesh, India: The Librarian, Vikaram University Library.

Union, New Jersey, U.S.A.: Library, Newark State College, Morris Avenue, Box 411.

Urbana, Illinois 61803, U.S.A.: Serials Division, Library, The University of Illinois at Urbana Champaign.

Utrecht, Holland: Bibl. Rijksuniversitiet, Willevrouwenstr. 11.

Utrecht, Holland: Institute for Iranian Studies, Heidelberglaan 2, Postbus 80118.

Vallabh Vidyanagar, Gujerat State, India: The Librarian, Sardar Patel University.

Vancouver, British Columbia, V6T 1W5, Canada: Serials Division, Main Library, University of British Columbia, 2075 Wesbrook Place.

Varanasi, 221005, India: Banaras Hindu University.

Varanasi, 221005, India: Post Graduate Institute of Medical Sciences, Library, Banaras Hindu University.

Vatican City, Rome, Italy: Monsignore Prefetto, Biblioteca Apostolica Vaticano.

Victoria, B. C., Canada: Serials Division, McPherson Library, University of Victoria.

Vienna, Austria: Gerold \& Co, 1 Graben 31, A-1011.

Villanova, Pa. 19085, U.S.A.: Falvey Memorial Library, University of British Columbia.

Visakmapatnam, India: D. V. S. Krishna Memorial Library, Andhra University.

Vittoria, Spain: Seminar Consiliar Diocesano.

Waco, Texas 76706, U.S.A.: Baylor University, Serials Dept., P.O. Box 6307.

Wake Forest, North Carolina, 27587, U.S.A.: South Eastern Baptist Theological Seminary Library, P.O. Box 752.

Waltham 64, Mass., U.S.A.: Brandeis University Library.

Washington, D.C. 20006, U.S.A.: George Washington University.

Washington, D.C. 20007 , U.S.A.: Georgetown University Library, Serials Department, 37 th \& 0 Streets, N.W.

Washington, D.C. 20016, U.S.A.: Periodicals Library, American University.

Washington, D.C. 20540, U.S.A.: The Library, Library of Congress, Order Division.

Washington, D.C. 20036, U.S.A.: The Library, Sydney R. and Elsa W. Mason Library, Johns Hopkins University, 1740 Massachusetts Avenue.

Washington, D.C. 17, U.S.A.: Semitics Library, Room 318 Mullen, Catholic University of America.

Washington, 99163, U.S.A.: Humanities Library, Washington State University Library, Pullman.

Washington, D.C. 20007, U.S.A.: Mrs. L. S. Anberry Rahardja, 3508, Prospect Street, N.W.

Wellingborough, Northants NN8 2QT: Collet's Subscription Export Dept, Denington Estate.

Wellington, New Zealand: The Library, (Periodicals), University of Wellington, Private Bag.

Wiesbaden, Germany: Otto Harrassowitz, Taunusstrasse 5, P.O. Box 2929, D-6200.

Williamsburg, Va. 23185, U.S.A.: Periodicals Department, Earl Gregg Sven Library, College of William and Mary.

Windsor, Canada: University of Canada.

Winnipeg, Manitoba R3Z 2N2, Canada: University of Manitoba, Elizabeth Dafoe Library, Periodicals Department.

Winston-Salem, N. Carolina 27109, U.S.A.: Wake Forest University Library, P.O. Box 7777 , Reynolds Station.

Würzburg 87, Germany: Bibliothek der Universität, Domerschulgasse 16. 
York, YO1 5DD, England: Librarian, University of York Library, Heslington.

Yoshida Yamaguchi, 753 Japan: Yamaguchi Daigaku, Fuzoku Toshokan (B).

Zaria, Nigeria: The Librarian, Kashim Ibrahim Library, Ahmadu Bello University. Zürich, Switzerland: Zentralbibliothek, Zahringerplatz 6, CH 8025. 


\section{NOTICE TO CONTRIBUTORS}

All editorial communications should be addressed to the Secretary, Royal Asiatic Society, 56 Queen Anne Street, London W1M 9LA, in the first instance.

Contributions submitted for publication in the Society's Journal should be typed in double spacing on A4 size paper, leaving a margin of at least $30 \mathrm{~mm}$. at the left-hand side. Sheets should be numbered consecutively at the top righthand corner. Notes, numbered consecutively through the article, should be typed on a separate sheet or sheets at the end and not at the foot of each page.

Authors are urged to study the conventions employed in recent issues of the Joumal, particularly the style of citation of books and periodical articles, and the bibliographical information inserted at the head of reviews. Contributions should be submitted in a form ready for the printer; later corrections, whether involving over-running or not, can be accepted only at the Editor's discretion and will incur a charge to the author. Authors will receive page proofs; galleys will not normally be sent.

Authors of articles will be supplied with 25 free copies of offprints. Additional copies can be obtained on request at moderate charges; authors are asked to notify the Editor of their requirements when returning proofs. 


\section{MEMBERSHIP AND SUBSCRIPTION}

1. Any person desirous of becoming a Fellow must be nominated by one Fellow and seconded by another, of whom one must act on a personal knowledge that the candidate is likely to be a suitable and useful Fellow; and the nominating Fellows shall address the Secretary in writing and give the candidate's name, address, and occupation, or status, and shall state to which class the candidate desires to be admitted. Provided always that for persons domiciled abroad it shall be within the power of the Council, on being satisfied as to the credentials of any particular candidate, to dispense with these conditions.

2. The annual subscription for each class of Fellow is:-

Fellows resident in the United Kingdom . . . . . . . . 10.00

Fellows subscribing under covenant $\ldots \ldots \ldots \ldots \ldots \ldots \ldots . \ldots . \ldots$

Fellows residing abroad $\ldots \ldots \ldots \ldots \ldots \ldots \ldots \ldots$

3. Library Associates, elected for five years only . . . . . . . . 3.00

Libraries . . . . . . . . . . . . . . . . . . . . . . . 15.00

4. The first payment of subscription is due on election, but if a candidate be elected in November or December of any year, the first annual subscription paid by him shall cover the year beginning on the 1st January next after his election.

5. Annual subscriptions shall be due on the first day of January of each year. Note: Payments by Deed of Covenant can be made only by Fellows who pay United Kingdom Income Tax. The attention of applicants residing abroad is drawn to the back cover of the Journal where the U.S. and Canadian dollar equivalents of these subscriptions are given.

P.T.O. 
When a candidate is proposed for election, this form should be completed in block letters and sent to THE SECRETARY, ROYAL ASIATIC SOCIETY, 56 QUEEN ANNE STREET, LONDON W1M 9LA.

\title{
ROYAL ASIATIC SOCIETY
}

\author{
CERTIFICATE OF RECOMMENDATION
}

Name

Occupation

Address .

Being desirous of becoming a Fellow of the ROYAL ASIATIC SOCIETY, we being

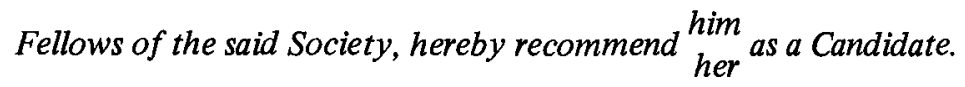

Proposer

Address

Seconder

Address.

Date 


\section{Oxford University Press}

Churchill, Cripps, and India 1939-1945

\section{R. J. Moore}

This is the story of a missed opportunity. Professor Moore argues that Sir Stafford Cripps's mission to India in 1942 could, if successful, have prevented the ultimate partition of India, and that its failure can be attributed to the baleful influence of Churchill, acting in support of the over-cautious and often stubborn Viceroy, Lord Linlithgow. $£ 7.50$

\section{The Beginning of the Great Game in Asia 1828-1834}

\section{Edward Ingram}

This book explains why the British became worried by the expansion of Russia, why they were right to be worried, and how they tried to safeguard British India without entangling imperial defence with the balance of power in Europe. $£ 11$

\section{Rajah Charles Brooke: Monarch of all he Surveyed}

\section{Colin N. Crisswell}

Here is the first full-length biography of Charles Brooke, who ruled as Rajah of Sarawak from 1868 until 1917. He was eccentric and strongminded, and he formalized the administration and finances of Sarawak. The author has studied all the known source material on this hitherto obscure figure. Illustrated $£ 11.50$

\section{Sacred and Profane Dimensions of Love in Indian Traditions}

The Gitagovind of Jayadeva

\section{Lee Siegel}

This is an inquiry into the conceptions of love in its sacred and profane dimensions as a universal theme in religious and secular literature. The author examines the way in which the generic experience finds expression in Indian traditions, and the way in which those traditions are exemplified in the twelth-century Sanskrit poem, the Gitagovind. f12.25 


Microfilmed copies
of back numbers
of the
JOURNAL OF
THE ROYAL ASIATIC SOCIETY
are available from
E.P. MICROFORM LIMITED
EAST ARDSLEY
WAKEFIELD WF 3 2JN
YORKSHIRE




\section{- Newbooks from CAMBRIDGE \\ The Cambridge History of China}

Volume 3: Sui and T'ang China, 589-906, Part I

Edited by DENIS TWITCHETT

The second volume of the History to be published covers the second great period of unified imperial power when China established herself as the centre of a wider cultural sphere embracing Japan, Korea and Vietnam.

$£ 35.00$ net

Forthcoming

CAMBRIDGE STUDIES IN CHINESE HISTORY, LITERATURE AND INSTITUTIONS

\section{The Aristocratic Families of Early Imperial China}

A case Study of the Po-ling Ts'ui Family

PATRICIA BUCKLEY EBREY

This study of the Po-ling Ts'ui family (or clan) from the end of the second to the end of the ninth centuries demonstrates the fluctuation in aristocratic influence and the changing basis of such families' prestige and power.

f11.50 net

CAMBRIDGE STUDIES IN CHINESE HISTORY, LITERATURE AND INSTITUTIONS

\section{Land and Lineage in China}

A study of T'ung-ch'eng county, Anwhei, in the Ming and Ch'ing Dynasties

HILARY J. BEATTIE

An examination of landholding, taxation and social structure in one county of central China which became famous in the Ming and Ch'ing periods for producing great officials and remarkable intellectual traditions.

$£ 12.50$ net

\section{Paperback edition}

\section{Japan, China and the Modern World Economy}

Toward a Reinterpretation of East Asian Development c. 1600 to c. 1918

\section{FRANCES V. MOULDER}

'This work is a provocative and valuable contribution to the literature... Though the debate about the early developmental efforts of China and Japan will hardly be stopped, this study will make it more productive.'

\section{CAMBRIDGE UNIVERSITY PRESS}




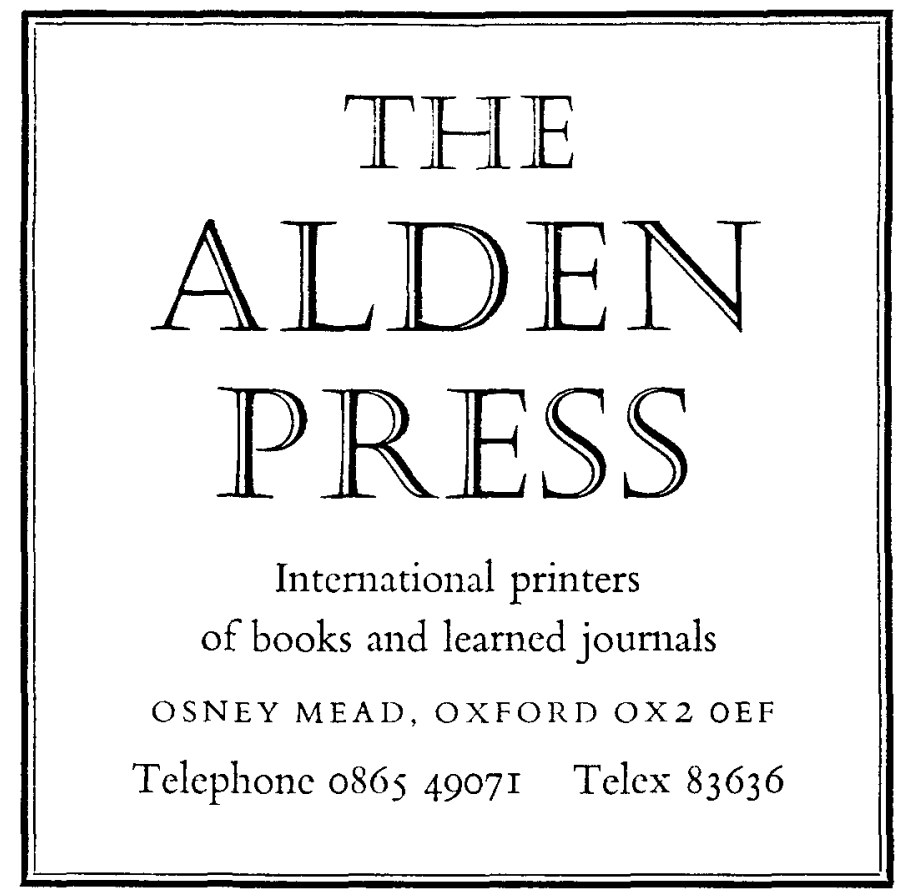




\section{new from Undena}

\section{Biobibliography of Ismā'i ili Literature}

By Ismail K. Poonawala - Studies in Near Eastern Culture and Society, vol. 1, pp. xx-533, $\$ 40.00$ (hard cover).

A comprehensive Ismã ili biobibliography listing more than 200 authors and 1,300 titles with locations of existing manuscripts based on Ismã îli and non-Ismă îili work.

\section{Ebla: Recent Discoveries at Tell Mardikh}

By P. Matthiae - MANE 1/6, 16 pp., 26 plates, $\$ 6.50$.

Ebla in the Period of the Amorite Dymasties and the Dynasty of Akkad. Recem Archaeological Discoveries at Tell Mardikh. Translation of a major article in Orientalia 1975 with many new illustrations.

\section{Terqa Preliminary Reports}

TPR 10: Fourth Season: Introduction and Stratigraphic Record, by G. Buccellati $B M$ 10. In press.

Evidence for a major urban settlement at the end of the fourth and the beginning of the third millennium, and the stratigraphic record of an important Khana period cuneiform archive.

For additional information, descriplive flyers of individual titles, and a general calalogue, write: UNDENa PUBliCations. P.O. Box 97. R.A.. Malibu, Ca 9026.5. 


\section{J.THORNTON \& SON}

FOR ALL BOOKS (BRITISH AND FOREIGN)

relative to the Languages, Literatures,

Antiquities and Archacology of

NEAR \& FAR EAST. EGYPT.GREECE AND ROME

and other books of an ACADEMIC NATURE

BOOKS PLRCHASED

ALL ENQUIRIES ANSWERED

11 BROAD STREET, OXFORD

Telephone: 42939

Cables: HORNBROOK, OXFORD 


\title{
ROYAL ASIATIC SOCIETY'S PUBLICATIONS
}

\author{
Sole Agent: Messrs. E. J. Brill (LEIDEN), 41 Museum St., London WC1.
}

\section{Oriental Translation Fund New Series}

(21) RUST'RAVRLI (SEOT'Bu). The Man in the Panther's 8kin. A romantic epic. (Trans.) from the Georgian by
M. 8. Wardrop (1912). Reprinted 1967.

(28) VISRAMTANI. The story of the loves of Vis and Famin. Trans. from the Georgian version by $O$. Wardrop Ramin. Trans. from the Georgian version by 0. Wardirop
(1916). Reprinted 1967 .

(28) AX-MUHAssIN. The Table-Talk of a Mesopotamian Judge (Trans.) from “ Ninhwar al-Mnhadarah" by D. 8 Margo (Trans.) from “ Nishwar al-Muhadarah by
M. D.75.

(29) AI-BXBU I-HXDI'ASHAR. A Treatise on the Princtples of Shit to Theology. By Hasan B. Y Asuf B. AIt Principles of Bhr'ite Theology, By Hasan B. Y Asuf B. 'An Ibnn'1-Mutahhar Al-Hili. With Commentary by Midid-jFidil Al-Hill. (Trans.) William MeElwee Miller. (Reprinted),
1958.

(30) HAMDULI.AF AI-MUSTAUTI AK-QAZWINI. The Zoological Bection of the Nushstu-l-Qulub. (Ed.) J.
Stephenson. 1928.

(31) VICTTRAKARNIKAVXDXNODDHRTA. A Collection

of Buddhistic legends. Nevtri text. (Ed. and trans.) Hans
Jorgensen. 1931. Jorgensen. 1931 .

(38) VIDYAPATI THAKURA. Paraha-Parlksha, or The Test of Man. (Trans.) Blr G. A. Grierson. 1935.14 .75
(84) IBN ABI'I-DUNYA \& MAJD AIRDN ALTCSI ALGHAZXLF. Tracta on Listenfng to Musle. Being a transiation of "Dhamm al-malahi" and "Basoariq al-ilma." (Bd.) J. Robson. Text in Arabic. 1938. Basariq at-hma. (36) SFAR MALWASIA. D.C.81. The Book of the Zodine. Trans. and notes by $\mathrm{B}$. 8 . Drower with a facaimlle of the original Mandaic text. 19i9. (87) THOMAS (F. W.) (Ed, and Transiator). Tibetan Literary texts and documents concerning Chinese Turkestan. Vol. 2. Documents. 1951. $\$ 110 \cdot 50$

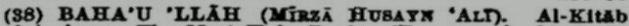
al-Aqdas or The Moet Holy Book. (Trans and ed.) E. E. Elder and W. MeE. Miller. 1961. Sewn £1.50 (89) AL-RAKTM ABU ABDULLAF MUHAMMAD B. 'ABDULLAF AI-NAISABURI. Introduction to the Science of Tradition, being AJ-madkhal ila ma'rifat al-Ikli,, (ed.) trans.. introduction and notes by James Robson. 1958. $12 \cdot 75$ (40) TEOMAS (F. W.) (Ed. and Tranalator). Tibetan Literary texts and documents concerning Chinese Turkestan. Selected
and translated into English. Vol. 3. 1955.
i5.00 (41) - Vol. 4. Indices.

(42) TIBBETTS (G.R.) Arab Navigation in the Indlan Ocean before the coming of the Portuguese. 1972. 43) CAMERON (A. J.) Abu Dharr al-Ghifari. 1974. $25 \cdot 80$

\section{Prize Publication Fund}

(4) BRA Y (D. DB 8.) The Life-history of a Brahul. 1918. s. 00

(11) GRIERSON (SrR G. A.) Torwall. An Account of a Dardic Language of the Swat Kohlstan. 1929. \&5.00

(16) TA QIZADEH (S. H.) The Early Iranian Calendars. 1938. $\mathbf{1 1 \cdot 7 5}$
(19) RICHARDSON (F. R.) Ancient Historical Edicts at Lhasa, and the Mu Trung/Khri Gteug Lde Brtasn Treaty of Lhasa, and the Mu Trung/Khrl Gteug Lde Brtasn Treaty of
A.D. 821-822 from the Inscription at Lhasa. 1952. I2.00 (20) CLA USON (SIR GRRARD). Turkish and Mongolian Studies. 1988. £. 00

(21) FDMONDS (C. J.) A Pilgrimage to Lallsh. 1967. \&s.00

\section{James G. Forlong Fund}

(6) HASAN (HADI). Falakd 1-Shirwani, His Times, Lfe and Works. 1929. (9) FAKA KI-8HiRW WANI. Diwan. Persian Text (ed.) $\underset{£ 2 \cdot 50}{\text { Had }}$ (10) JUWAYNI. Ta'rlkh-1-Jahs̃n-Gnahsy of Juwaynt. Vol. III. (Introduction by $81 \mathbf{E}$ E. Denison Ross.) 1931 . $£ 2 \cdot 50$ (11) ROSS (E. DExtsox) \& WINGATE (R. O.) (Ed.) Dialogues in the Eastern Turkd on Subjects of Interest to Traveliers. 1934.

(14) DAVE (T. N.) A Study of the Gujarati Language In the XVth Century. 1935.

(15) READ (A. F. C.) Balti Grammar. 1934.

$\$ 2 \cdot 50$

(26) MANIPATI-CA RITA (Two PRARrit Versions OF THE). Introduction, romanlzed text and translation. (Ed.) $\mathbf{R}$ Willams. 1959. 今8. 50 (27) OKELL (J.) Guide to the romanization of Burmese. 1972.

\section{Monographs}

(2) WINTERNITZ (M.) Catalogue of South Indian Sanskrit
1ss. belonging to the R.A.8. 1902.
$£ 8.00$ (11) SAYCE (A. H.) and PINOHES ('T. G.) The Tablet from Yuxgat in the Liverpool Institute of Archieology. 1907. $\varepsilon_{2} \cdot 00$

(28) FIBCHEL (W. J.) Jews in the Economic and Polltical Lffe of Medlaeval Islam (1937). Reprinted 1968 . $\& 3 \cdot 25$

(26) MLNORSKY (V.) Persia in A.D. 1478-1490. An abridged translation of Fadiulish b. Razbihan KhunI's Trish-s 'Rlam-ấra-yi Amini. 1957. £3-25

(27) ELFEN BEIN, J. H. The Baluchl Language. A Dlalectology with Texts. 1966.

$\varepsilon 2 \cdot 00$

Dr. B. C. Law Trust Fund

CHI (R.8.Y.) Buddhist Formal Logic. 1969. £5.00
OATA LOGU E OF PRINTED BOOKS published before 1992. in the LJbrary of the Royal Aslatic Soctety. 1940 [B].
Centenary Supplement

$\mathbf{E 7} \cdot 00$ 


\section{ROYAL ASIATIC SOCIETY}

56 Queen Anne Street, London W1M 9LA (Tel. : 01-935 8944)

Fellows' Annual Subscriptions:-

(a) Fellows resident in the United Kingdom, £10.00. (Fellows paying U.K. income tax may subscribe under covenant at $£ 9.00$ per annum.)

(b) Fellows residing abroad, $£ 8.00$, or U.S.\$21, or Can.\$24.

(c) Library Associates elected for five years only, $£ 3.00$, or U.S.\$8, or Can.\$9.

Journal Subscriptions:--

Post free to Libraries or non-members, $£ 15.00$, or U.S.\$40, or Can.\$45 per annum.

Payment in advance to the Secretary.

The price of each half-yearly issue is pro rata.

Payment of Subscriptions may be by:-

(a) Cheque

(b) Bank Draft

(c) International Money Order

N.B. Dollar amounts quoted allows for exchange and for collection charges.

\section{THE JOURNAL}

The Society's Journal contains original articles on the archaeology, art, history, language, literature, beliefs, and customs of the East.

Acknowledgement of books and pamphlets presented to the Society is made in the Journal, which reviews the more important, where possible, but cannot return those not reviewed.

PRINTED IN GREAT BRITAIN AT THE ALDEN PRESS, OXFORD 